\title{
Single-atom switching in photonic crystals
}

\author{
Marian Florescu and Sajeev John \\ Department of Physics, University of Toronto, 60 St. George Street, Toronto, Ontario, Canada M5S 1A7
}

(Received 1 November 2000; revised manuscript received 14 May 2001; published 2 August 2001)

\begin{abstract}
We describe the role of first non-Markovian corrections to resonance fluorescence in photonic crystals, using a perturbative expansion of the Heisenberg equations of motion in powers of the atom-field reservoir coupling strength. Non-Markovian effects arise from the rapid variation of the photonic density of states with frequency. Our method recaptures the physics of the photon-atom bound state in the presence of a full photonic band gap. For the anisotropic three-dimensional photonic band gap, it predicts remarkable features in the resonance fluorescence, such as atomic population inversion and switching behavior in a two-level atom for moderate values of the applied laser field. The magnitude of the switching effect depends sensitively on the external laser intensity and its detuning frequency from the atomic transition. The robustness of this effect against nonradiative decay and dephasing mechanisms is also investigated.
\end{abstract}

DOI: 10.1103/PhysRevA.64.033801

PACS number(s): 42.50.Gy, 42.50.Hz, 42.70.Qs

\section{INTRODUCTION}

Photonic crystals constitute a new class of dielectric materials, in which the basic electromagnetic interaction is controllably altered over certain frequency and length scales. In photonic crystals, the synergetic interplay between the microcavity resonances of individual dielectric particles and the Bragg scattering resonances of the dielectric array [1] leads to the formation of a photonic band gap ( $\mathrm{PBG}$ ), a range of frequencies for which no propagating electromagnetic modes are allowed $[2,3]$. The presence of the photonic band gap in the dispersion relation of the electromagnetic field gives rise to new phenomena, including the inhibition of the spontaneous emission [4], strong localization of light [5], formation of atom-photon bound states [6], collective switching behavior, and atomic inversion without fluctuations [7]. Photonic crystals represent the ultimate example of a confined photonic system [8-11], in which the photonic density of states (DOS) is suppressed throughout the volume of the material over a certain frequency window.

These features open the possibility for important technological applications of photonic crystals: low-threshold microlasers, ultrafast optical switches, all-optical transistors, and memory devices, to name a few. Previous studies $[12,13]$ suggest that a laser operating near a photonic band edge may posses unusual spectral and statistical properties, as well as a low-input-power lasing threshold due to the fractional inversion of the atoms in the steady-state limit. In certain conditions, a doped photonic crystal exhibits optical bistability in the atomic response to an applied laser field [14]. Furthermore, the optical bistability may occur at very low intensities of the external laser field and relatively small densities of impurity atoms. Also, the atomic population inversion and the statistics of a collection of atoms driven by a laser field in a photonic crystal show remarkable features [7]. For large deviations in the photonic-mode density, strong atomic population inversion has been predicted. When the density of impurity atoms is high enough, the atoms collectively switch from the ground state to the excited state at a very-welldefined threshold value of the applied laser-field intensity.

However, rapid variations in the photonic DOS with frequency leads to major modifications of the quantum optics in confined photonic materials relative to an ordinary vacuum. The commonly used Markov approximation relies on the basic assumption of a smoothly varying DOS of a photonic reservoir of modes, which is severely violated in a highrefractive-index photonic crystal [15]. In certain models of the photonic crystals, the widely used Born approximation is inadequate to describe the strong atom-field interaction near a divergence in the photonic DOS [6].

A perturbation theory was developed by Wódkiewicz and Eberly (WE) [16] and applied to investigate the nonMarkovian character of the Bloch equations for the two-level atom coupled the radiation reservoir of ordinary vacuum. A similar approach has been used in the context of a structured radiation reservoir by Lewenstein et al. [17,18]. From a strict mathematical point of view, a quantum mechanical system whose energy spectrum in bound from below cannot spontaneously decay in a purely exponential manner [19]. For such systems, the Paley-Wiener theorem [20] requires that in the long-time limit the decay be slower than exponential. Several theoretical studies [21-23] have shown that the decay behavior has in fact a nonexponential tail (for large times the traditional exponential is replaced by a slower decay as $t^{-2}$, in agreement with the combined requirements of energy positivity and the Paley-Wiener theory). However, the size of the nonexponential contribution is of unobservable magnitude for a two-level atom coupled to the radiation reservoir of free space. The WE perturbation technique also gives a general solution to the two-level atom resonance fluorescence problem and reproduces the results of Torrey [51], Mollow [52], and Heitler [53] in the appropriate limits.

In this paper, we use a perturbative technique based on the Heisenberg equations of motion approach to describe the first-order non-Markovian corrections to the spontaneous emission and the resonance fluorescence phenomena in photonic crystals. The perturbation parameter is the coupling strength between the atomic system and the field reservoir of modes. A second-order expansion in this coupling parameter is equivalent to the Born approximation.

The paper is organized as follows. In Sec. II, we formulate the problem of spontaneous emission in the context of frequency-dependent photonic reservoirs and present some 
specific models for the spectral density of photonic band gap materials. In Sec. III, we introduce a perturbative technique based on the Heisenberg equations of motion approach and analyze the first-order non-Markovian corrections to the spontaneous emission. In Sec. IV, we solve the problem resonance fluorescence in PBG materials in the second-order of the perturbation approach. We show that the atomic system can exhibit switching behavior as a function of the laser-field intensity and laser-field frequency. We also analyze the dissipative effects of dipole dephasing interactions and nonradiative relaxation of the atomic system.

\section{OPTICAL-MODE DENSITY NEAR A PHOTONIC BAND GAP}

We consider a two-level atom interacting with a quantized electromagnetic field of a photonic crystal. In the rotating wave approximation (RWA) and in a frame of reference rotating with the atomic resonance frequency $\omega_{A}$, the Hamiltonian describing the total system is

$$
\begin{gathered}
H=H_{0}+H_{\text {int }}, \\
H_{0}=\sum_{\lambda} \hbar \Delta_{\lambda} a_{\lambda}^{\dagger} a_{\lambda}, \\
H_{\text {int }}=i \hbar \sum_{\lambda} g_{\lambda}\left(a_{\lambda}^{\dagger} \sigma_{12}-\sigma_{21} a_{\lambda}\right),
\end{gathered}
$$

with

$$
g_{\lambda}=\frac{\omega_{A} d_{21}}{\hbar}\left[\frac{\hbar}{2 \epsilon_{0} \omega_{\lambda} \mathcal{V}}\right]^{1 / 2} \mathbf{e}_{\lambda} \cdot \mathbf{u}_{d},
$$

and $\sigma_{i j}=|i\rangle\langle j|(i, j=1,2)$ are the atomic pseudospin operators, $\sigma_{3}=\sigma_{22}-\sigma_{11}$ describes the atomic inversion, $a_{\lambda}$ and $a_{\lambda}^{\dagger}$ are the radiation-field annihilation and creation operators, and $\Delta_{\lambda}=\omega_{\lambda}-\omega_{A}$ represents the $\lambda$-mode detuning frequency from the atomic frequency. Here, the atomic dipole moment $\mathbf{d}$ has been chosen real, with $d_{21}$ its magnitude and $\mathbf{u}_{d}$ the unit vector. $\mathbf{e}_{\lambda} \equiv \mathbf{e}_{\mathbf{k}, \sigma}$, with $\sigma=1,2$, are the transverse polarization vectors of the radiation field, and $\mathcal{V}$ is the quantization volume.

We consider an effective mass approximation to the full dispersion relation of a photonic crystal. Within this approximation, two models for the near-band-edge dispersion are used. The first one is the isotropic model, which is obtained by symmetrizing the dispersion relation to all directions in $\mathbf{k}$ space. The $\mathbf{k}$ dependence of the frequency is given then by $\omega(\mathbf{k})=\omega(|\mathbf{k}|)=\omega_{C} \pm A\left(k-k_{0}\right)^{2}$, where $k_{0}$ is the radius of the sphere in $\mathbf{k}$ space about which we perform the expansion, and $A$ is a constant that depends on the specific photonic crystal considered. The plus (minus) sign stands for the case when the frequency is expanded about the upper (lower band edge). If we consider a photonic crystal with a large band gap $\left(\omega_{\text {gap }} \gg 2 c^{2} / A\right)$ and the atomic frequency nearly resonant with the upper band edge $\left(\omega_{C}\right)$, the influence of the lower band edge can be completely neglected [13]. Under these assumptions, the dispersion relation is $\omega(k)=\omega_{C}$ $+A\left(k-k_{0}\right)^{2}$. The density of states generated by this disper- sion relationship becomes divergent in the band-edge vicinity: $\rho(\omega)=\Sigma_{\mathbf{k}} \delta(\omega(\mathbf{k})-\omega) \approx 1 / \sqrt{\omega-\omega_{C}}$. As shown earlier [12], the isotropic model overestimates the phase space occupied by the band-edge photons of vanishing group velocity.

For a real dielectric crystal with an allowed point-group symmetry, the band edge occurs at certain points along the Bragg planes of the lattice. We will consider now a more realistic model, in which the dispersion relation is anisotropic. Within the effective mass approximation, this is defined by $\omega(\mathbf{k})=\omega_{C}+A\left(\mathbf{k}-\mathbf{k}_{0}\right)^{2}$, where $\mathbf{k}_{0}$ is a point of the Brillouin zone boundary. The density of states is now given by

$$
\begin{aligned}
\rho(\omega) & =\sum_{\mathbf{k}} \delta(\omega(\mathbf{k})-\omega)=\int_{\mathbf{S}_{\omega}} \frac{d S}{4 \pi^{3}} \frac{1}{\hbar\left|\nabla_{\mathbf{k}} \omega(\mathbf{k})\right|} \\
& \propto\left(\omega-\omega_{C}\right)^{d / 2-1},
\end{aligned}
$$

where $\mathbf{S}_{\boldsymbol{\omega}}$ is a surface of constant energy $\omega$ and $d$ represents the dimensionality of the photonic crystal. When the gradient of $\omega(\mathbf{k})$ vanishes, the integrand in the density of states (2.3) diverges. In a one-dimensional crystal, $\rho(\omega)$ becomes divergent as $\omega$ approaches $\omega_{C}$ (a square root divergence). For a two-dimensional photonic crystal, the divergence of the integrand gives rise to a step discontinuity in $\rho(\omega)$. In three dimensions (3D) the singularity becomes integrable, yielding finite and continuous values for the density of states. However, it does result in divergences of the slope (Van Hove singularities [24]) of the density of states $d \rho / d \omega$.

Photonic band gap materials typically consist of a two interpenetrating dielectric components. The first is a connected high-dielectric-constant backbone, and the second is a connected low-dielectric-constant network. In the microwave regime, drilling cylindrical holes in a bulk dielectric material along a well defined spatial mesh (length scale of the order of $\mathrm{mm}$ ) [25] or layer-by-layer fabrication techniques [26] can provide band gaps of $20 \%$ of the central frequency of the gap [25]. As a result of the length scales involved, in the IR and visible regimes one has to use microlithographic methods or self-ordered systems, such as inverted synthetic opals, in order to create a photonic band gap material. A rich variety of photonic crystal structures have been proposed and fabricated (silicon wafer structure [27], air spheres in a $\mathrm{TiO}_{2}$ dielectric backbone [28], $\mathrm{SiO}_{2}$ spheres in a InP backbone [29], air spheres in a CdSe nanocrystals backbone [30], etc.). The most recent breakthrough is a 3D photonic crystal with a 5\% complete 3D photonic band gap centered near $1.5 \mu \mathrm{m}$ [31]. The active "two-level atoms" in our model calculation can be embedded in the dielectric backbone or may be laser cooled and trapped in the void regions of the PBG material.

Our perturbation approach is based on the weak coupling between the atomic system and the radiation reservoir. Using the expression of the coupling constant (2.2) (see Appendix A for details and also Ref. [32] for an accurate description of atomic decay in cavities and material media) and appropriate values for the parameters that characterize the photonic crystals in the optical regime $\left(\omega_{A} \approx 10^{15} \mathrm{~Hz}, d_{21} \approx 10^{-29} \mathrm{C} \mathrm{m}\right.$, $\mathcal{V}=a^{3} \approx 10^{-18} \mathrm{~m}^{3}$, with $a$ the linear dimension of the unit 
cell of the photonic crystal), we have $10^{-6} \omega_{A} \leqslant g_{\lambda}$ $\leqslant 10^{-4} \omega_{A}$, a regime in which a perturbation treatment of the interaction of the atomic system with the radiation reservoir of the photonic crystal is justified. For simplicity, we follow the usual perturbation method, introduce an expansion parameter $\Lambda$, and rewrite $H_{\text {int }} \rightarrow \Lambda H_{\text {int }}$.

On the other hand, Eq. (2.1c) involves a sum over the transverse polarizations and integration over k space. Qualitatively, for an isotropic reservoir,

$$
H_{i n t}=i \hbar \Lambda \int_{0}^{\infty} d \omega \rho(\omega) g(\omega)\left(a_{\omega}^{\dagger} \sigma_{12}-\sigma_{21} a_{\omega}\right) .
$$

In order to apply a perturbational approach, the density of states should not become too large over the spectral range of interest. In an ordinary vacuum, perturbation theory is very realistic and it recaptures the main results of the two-level atom quantum optics [16]. We use the ordinary vacuum density of states $\rho(\omega) \propto \omega^{2}$ as a reference case for future discussions.

For the isotropic model, the interaction between the atomic system and the radiation reservoir becomes very strong near the band edge (the density of states is divergent for $\left.\omega=\omega_{C}\right)$. As a result, the perturbation theory results are qualitatively different from the exact solution. This, however, is an artifact of the isotropic model. For the anisotropic model, on the other hand, $\rho(\omega)$ remains finite over the entire spectral range of interest. Indeed, perturbation theory recaptures the non-Markovian effects associated with a rapidly varying density of states (the non-Markovian effects are most important near the band-edge frequency, corresponding to a spectral range determined by small values of the atomic detuning $\left.\Delta_{A C} \equiv \omega_{A}-\omega_{C}\right)$. In this spectral range, the anisotropic photonic crystal is a striking example of non-Markovian dynamics in the context of the Born approximation.

\section{SINGLE-ATOM SPONTANEOUS EMISSION}

The Heisenberg equations of evolution for the $\lambda$ mode of the radiation field, the atomic system polarization $\sigma_{12}$, and the atomic excitation population $\sigma_{22}$ are

$$
\begin{gathered}
\frac{d}{d t} a_{\lambda}(t)=-i \Delta_{\lambda} a_{\lambda}(t)+\Lambda g_{\lambda} \sigma_{12}(t), \\
\frac{d}{d t} \sigma_{12}(t)=\Lambda \sigma_{3}(t) \sum_{\lambda} g_{\lambda} a_{\lambda}(t), \\
\frac{d}{d t} \sigma_{22}(t)=,-\Lambda \sigma_{21}(t) \sum_{\lambda} g_{\lambda} a_{\lambda}(t)+\text { H.c. }
\end{gathered}
$$

We formally eliminate the field operators by integrating their evolution equation and substituting the result into the equations of motion of the atomic operators ("adiabatic" elimination of the field variables). We obtain

$$
a_{\lambda}(t)=\eta_{\lambda}+\Lambda \int_{0}^{t} d t^{\prime} G_{\lambda}\left(t-t^{\prime}\right) \sigma_{12}\left(t^{\prime}\right)
$$

where $\eta_{\lambda}=a_{\lambda}(0) e^{-i \Delta_{\lambda} t}$ is the vacuum contribution, the remainder is the source (atomic system) contribution, and $G_{\lambda}\left(t-t^{\prime}\right)=g_{\lambda} \exp \left[-i \Delta_{\lambda}\left(t-t^{\prime}\right)\right]$. This yields

$$
\begin{aligned}
\frac{d}{d t} \sigma_{12}(t)=\Lambda \sigma_{3}(t) & \eta(t)+\Lambda^{2} \int_{0}^{t} d t^{\prime} G\left(t-t^{\prime}\right) \sigma_{3}(t) \sigma_{12}\left(t^{\prime}\right), \\
\frac{d}{d t} \sigma_{22}(t)= & -\Lambda \sigma_{21}(t) \eta(t)-\Lambda^{2} \int_{0}^{t} d t^{\prime} \\
& \times G\left(t-t^{\prime}\right) \sigma_{21}(t) \sigma_{12}\left(t^{\prime}\right)+\text { H.c. }
\end{aligned}
$$

where $\eta(t)=\Sigma_{\lambda} g_{\lambda} \eta_{\lambda}(t)$ and the memory function is given by

$$
G\left(t-t^{\prime}\right)=\sum_{\lambda} g_{\lambda}^{2} \exp \left[-i \Delta_{\lambda}\left(t-t^{\prime}\right)\right]
$$

While the theoretical formulation used here is based on plane wave expansions of the radiation-field operators [inherited from the quantum electrodynamics of the free space, $\omega(\mathbf{k})=c|\mathbf{k}|$ ], the formalism can be easily generalized to incorporate realistic dispersion relationships and Bloch modes obtained through numerical band structure calculations (see Appendix A).

\section{A. Perturbation solution of the spontaneous emission problem}

We use the perturbation approach introduced by Wódkiewicz and Eberly [16] to solve the equation of motion of the atomic operators (3.2). In this regard, it is useful to explicitly include the expansion parameter $\Lambda$ in the evolution equations.

The nonlinear part of Eq. (3.2) arises entirely from the fact that the operator products in the integrand involve separate times $t \neq t^{\prime}$. In the Markovian approximation, the correlation time of the photonic reservoir is assumed to be very short compared to the time scale for a significant change in the atomic observables. Under this assumption, the memory function becomes [33] $G\left(t-t^{\prime}\right)=\left(\gamma_{21} / 2+i \delta_{21}\right) \delta\left(t-t^{\prime}\right)$, where $\delta_{21}$ and $\gamma_{21}$ are the usual Lamb shift and spontaneous emission rate, respectively.

In this approximation, the two-time operator product present in the equation of evolution is automatically linearized: $\quad \sigma_{3}(t) \sigma_{12}\left(t^{\prime}\right) \rightarrow \sigma_{3}(t) \sigma_{12}(t)=\sigma_{12}(t) \quad$ and $\sigma_{21}(t) \sigma_{12}\left(t^{\prime}\right) \rightarrow \sigma_{21}(t) \sigma_{12}(t)=\sigma_{22}(t)$. However, in the case of a photonic crystal with the density of states exhibiting rapid variations with frequency, the correlation time of the electromagnetic vacuum is not negligible on the time scale of the evolution of the atomic system, and the Markovian approximation scheme is inapplicable in general.

For a PBG material, a perturbation approach may be used to linearize the two-time operator product by collapsing it to an equal time product. This is done formally [16] by introducing the left Liouville operator of the system:

$$
\sigma_{i j}(t)=e^{-i L\left(t-t^{\prime}\right)} \sigma_{i j}\left(t^{\prime}\right)=\sum_{n=0}^{\infty} \frac{\left[-i\left(t-t^{\prime}\right)\right]^{n}}{n !} L^{n} \sigma_{i j}\left(t^{\prime}\right),
$$




$$
L^{n} \sigma_{i j}\left(t^{\prime}\right)=\hbar^{n}\left[\left[\ldots,\left[\sigma_{i j}\left(t^{\prime}\right), H\right], H\right], \ldots, H\right],
$$

where the left and right brackets in the commutator are repeated $n$ times. Equation (3.4) can be solved using the Laplace transform method. Before applying the Laplace transform, we drop all terms in the equations of motion that are higher than a given order in $\Lambda$. Following WE [16], we perform a perturbation expansion of the operator exp $[-i L \tau]$. The Liouvillian system can be written as $L=L_{0}$ $+L_{1}$, where $L_{0}$ is $O(1)$ and $L_{1}$ is $O(\Lambda)$. The Liouvillian operators $L_{0}, L_{1}$ are defined by $\hbar L_{0} \mathcal{O}(t)=\left[\mathcal{O}(t), H_{0}\right]$, $\hbar L_{1} \mathcal{O}(t)=\left[\mathcal{O}(t), H_{1}\right]$, with $\mathcal{O}(t)$ an arbitrary operator.

In the Born approximation, we keep contributions in the equations of motions up to the order $\Lambda^{2}$. This corresponds to replacing the Liouvillian $L$ with $L_{0}$ (associated with the noninteracting Hamiltonian $H_{0}$ ). Since the noninteracting Hamiltonian $H_{0}$ contains only photon operators, it will commute with the atomic operators. Consequently, $\sigma_{i j}(t)$ $=e^{-i L\left(t-t^{\prime}\right)} \sigma_{i j}\left(t^{\prime}\right) \approx e^{-i L_{0}\left(t-t^{\prime}\right)} \sigma_{i j}\left(t^{\prime}\right)=\sigma_{i j}\left(t^{\prime}\right)$.

We note that the zeroth order of perturbation does not always imply that the atomic operators remain unchanged in time. For example [16], consider the same problem in a nonrotating frame of reference, for which the free Hamiltonian is $H_{0}=1 / 2 \hbar \omega_{A} \sigma_{3}+\Sigma_{\lambda} \hbar \omega_{\lambda} a_{\lambda}^{\dagger} a_{\lambda}$. In the lowest order of perturbation (without the RWA), the atomic operators are given by $\sigma_{3}(t)=e^{-i L_{0}\left(t-t^{\prime}\right)}\left\{\sigma_{3}\left(t^{\prime}\right)\right\}=\sigma_{3}\left(t^{\prime}\right)$ and $\sigma_{ \pm}(t)$ $=e^{-i L_{0}\left(t-t^{\prime}\right)}\left\{\sigma_{ \pm}\left(t^{\prime}\right)\right\}=e^{ \pm i \omega_{A}\left(t-t^{\prime}\right)} \sigma_{ \pm}\left(t^{\prime}\right)$.

\section{B. Temporal behavior and steady-state solution}

In the Born approximation (the equations of motion are expanded up to the second order of the coupling constant), the Heisenberg equations of motion are

$$
\frac{d}{d t} \sigma_{12}(t)=\Lambda \sigma_{3}(t) \eta(t)+\Lambda^{2} \int_{0}^{t} d t^{\prime} G\left(t-t^{\prime}\right) \sigma_{3}\left(t^{\prime}\right) \sigma_{12}\left(t^{\prime}\right)
$$

$$
\begin{aligned}
\frac{d}{d t} \sigma_{22}(t)= & -\Lambda \sigma_{21}(t) \eta(t)-\Lambda^{2} \int_{0}^{t} d t^{\prime} \\
& \times G\left(t-t^{\prime}\right) \sigma_{21}\left(t^{\prime}\right) \sigma_{12}\left(t^{\prime}\right)+\text { H.c. }
\end{aligned}
$$

We average over the reservoir degrees of freedom, and consider the photonic reservoir to be initially in its vacuum state $\left[\left\langle a_{\lambda}^{\dagger}(0) a_{\lambda}(0)\right\rangle_{\mathcal{R}}=0,\left\langle\eta_{\lambda}\right\rangle_{\mathcal{R}}=\left\langle\eta_{\lambda}^{\dagger}\right\rangle_{\mathcal{R}}=0\right]$. The system of equations is further simplified by the identity $\sigma_{i j}\left(t^{\prime}\right) \sigma_{k l}\left(t^{\prime}\right)=\delta_{j k} \sigma_{i l}\left(t^{\prime}\right)$. For simplicity, hereafter we drop the superscripts $(a)$, associated with reservoir average $\left\langle\sigma_{i j}\right\rangle_{\mathcal{R}} \equiv \sigma_{i j}^{a}$, and set $\Lambda=1$. Under these assumptions, Eqs. (3.5) can be conveniently solved in operator form by making the following substitution:

$$
\begin{aligned}
& \sigma_{ \pm}(t)=\sigma_{ \pm}(0) \cdot S_{ \pm}(t), \\
& \sigma_{22}(t)=\sigma_{22}(0) \cdot S_{22}(t) .
\end{aligned}
$$

Here, $S_{ \pm}(t)$ and $S_{22}(t)$ are $c$ numbers. Introducing the Laplace transforms $\quad x(p) \equiv \widetilde{S}_{22}(p)=\mathcal{L}\left\{S_{22}(t)\right\}, \quad z(p)$ $\equiv \widetilde{S}_{12}(p)=\mathcal{L}\left\{S_{12}(t)\right\}, \quad \widetilde{G}(p)=\mathcal{L}\{G(t)\}, \quad$ and $\widetilde{G}^{*}(p)$ $=\mathcal{L}\left\{G^{*}(t)\right\}$, where $\widetilde{f}(p)=\mathcal{L}\{f(t)\}=\int_{0}^{\infty} e^{-p t} f(t)$, Eq. (3.5) yields

$$
\begin{gathered}
x(p)=\frac{S_{22}(0)}{p+\widetilde{G}(p)+\widetilde{G}^{*}(p)}, \\
z(p)=\frac{S_{12}(0)}{p+\widetilde{G}(p)} .
\end{gathered}
$$

The temporal evolution of the atomic variables (3.6) is then obtained immediately by evaluating the inverse Laplace transforms of these equations. Finally, we emphasize that the ansatz (3.6) allows us to solve the Heisenberg equations of motion in an operator form.

\section{Isotropic model}

In the isotropic model of a photonic crystal, the dispersion relation for a one-dimensional gap is extrapolated to all spatial dimensions. The infinity of modes available for the photon near the band edge causes an additional strong-coupling effect over and above that of the resonant interaction of the atom with photons whose group velocity $d \omega_{k} / d k$ vanishes (the energy bands have zero slope on a Brillouin zone boundary). The dressing of the atom by its own interaction becomes strong enough to split the atomic level by a considerable amount. A low-order perturbation approach leads to inaccuracies if the atomic transition frequency is tuned too close to the singularity in $\rho(\omega) \propto 1 / \sqrt{\omega-\omega_{C}}$. However, it is an appropriate formalism when the atomic resonant frequency is detuned slightly away from the divergence in $\rho(\omega)$.

Near a photonic band edge, the Born approximation provides a useful starting point for recapturing the photon-atom bound state solution. By selectively retaining leading nonMarkovian terms in the perturbation scheme, it is possible to recapture strong-coupling effects such as the vacuum Rabi splitting of the atomic level and the fractionalized steadystate inversion. This provides the basis for applying the same formalism to the case of a driven system near the band edge. We derive the occurrence of non-Markovian switching effects in the atomic excitation probability which occur at low threshold of the external cw laser field.

In the effective mass approximation and long-time limit $\left[\omega_{A}\left(t-t^{\prime}\right) \gg 1, p / \omega_{A} \ll 1\right]$, the memory function and its Laplace transform are given by (see Appendix B 1)

$$
\begin{gathered}
G_{I}\left(t-t^{\prime}\right)=\beta_{I}^{3 / 2} \frac{e^{-i\left[\pi / 4-\Delta_{A C}\left(t-t^{\prime}\right)\right]}}{\left(t-t^{\prime}\right)^{1 / 2}}, \\
\widetilde{G}_{I}(p)=\beta_{I}^{3 / 2} e^{-i \pi / 4} \sqrt{\frac{\pi}{p-i \Delta_{A C}}},
\end{gathered}
$$

where $\Delta_{A C}=\omega_{A}-\omega_{C}$ is the detuning of the atomic frequency with respect to the band-edge frequency $\omega_{C}$, and $\beta_{I}$ 


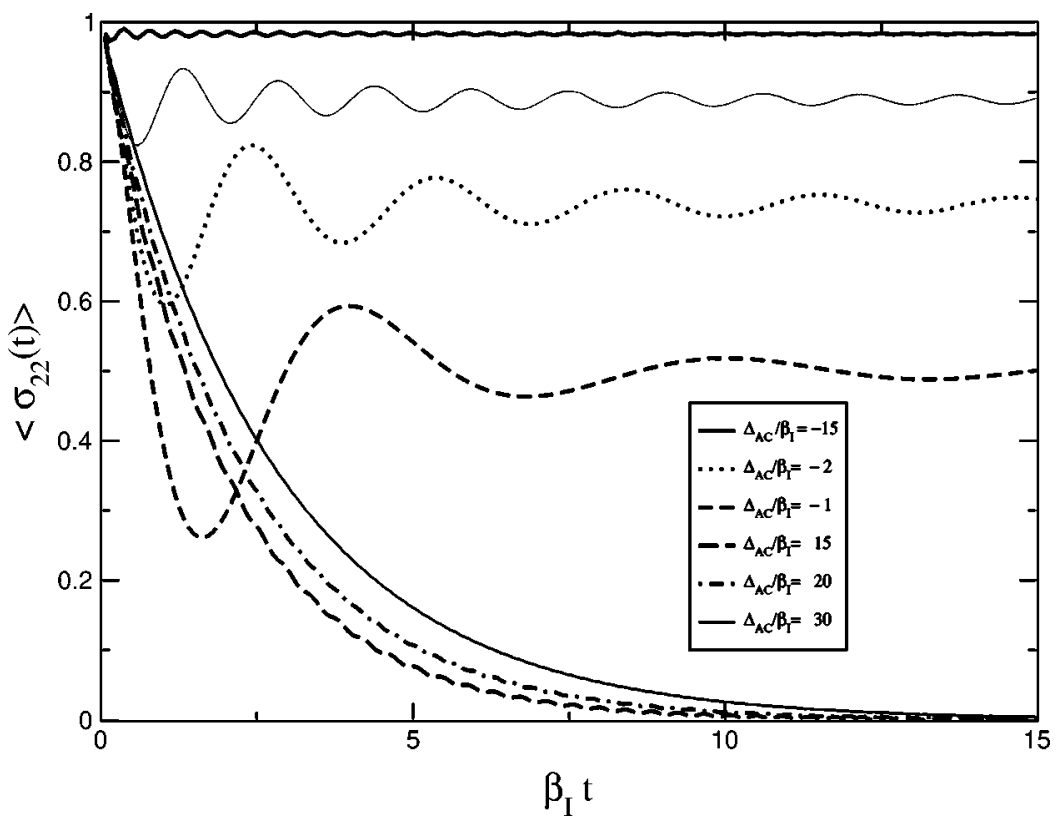

FIG. 1. Atomic population on the excited state, $\left\langle\sigma_{22}\right\rangle$, as a function of $\beta_{I} t$, for various values of the atomic detuning, from $\Delta_{A C} / \beta_{I}=-15$ for the thick solid curve to $\Delta_{A C} / \beta_{I}=30$ for the thin solid curve.

is a constant that depends on the nature of the band-edge singularity. For the isotropic model, it takes the form $\beta_{I}^{3 / 2}$ $=\omega_{A}^{7 / 2} d_{21}^{2} / 6 \hbar \epsilon_{0} \pi^{3 / 2} c^{3}$.

Using the explicit form for $\widetilde{G}_{I}(p)$ in Eq. (3.7), it is straightforward to evaluate the inverse Laplace transform and recapture the radiative dynamics described by Eq. (3.6). The details of this evaluation are given in Appendix C.

Figure 1 shows the atomic population on the excited state as a function of the scaled time for various values of the atomic detuning from the photonic band edge. Consistent with our perturbational approach, we scan the atomic frequency space, avoiding the positive detuning side of the spectral region surrounding the band-edge singularity, where the strong atom-radiation interaction (and a divergent density of states) make a low-order perturbation approach invalid.

Clearly, if the bare atomic level is placed inside the photonic band gap, our approach (in its domain of validity) is able to recover the splitting of the atomic level into dressed states caused by the interaction between the atomic system and its own radiation. However, when the bare atomic level is outside the gap, the photon-atom bound-state component suggested by earlier approaches [6,12] cannot be recaptured in a low-order perturbation analysis. In our approach, outside the gap the atomic population vanishes in the long-time limit, regardless how close the atomic frequency is to the band-edge frequency. On the other hand, the occurrence of a nonzero steady-state excitation amplitude for a level outside the gap appears to be an artifact of the isotropic model (divergent) density of states, which cannot be recaptured by a low-order perturbation expansion. For the more realistic anisotropic model of a PBG material dispersion relation, the steady-state excitation probability vanishes at the band edge for both the variational wave-function method and the nonMarkovian perturbation approach. However, Rabi splitting can be achieved for an extremely low applied laser field.

\section{Anisotropic model}

For a real dielectric crystal with an allowed point-group symmetry, the band edge occurs at certain points along the
Bragg planes in wave-vector space. We consider now a more realistic model, in which the dispersion relation is anisotropic. In the effective mass approximation, the dispersion is given by $\omega(\mathbf{k})=\omega_{C}+A\left(\mathbf{k}-\mathbf{k}_{0}\right)^{2}$, where $\mathbf{k}_{0}$ is a point of the Brillouin zone boundary. In this section, we analyze a threedimensional photonic crystal, characterized by a continuous and finite density of states $(2.3)\left[\rho(\omega) \propto\left(\omega-\omega_{C}\right)^{1 / 2}\right]$. Although there is a spectral region over which the density of states of a three-dimensional photonic crystal becomes larger than the ordinary vacuum density of states, we identify several spectral regions over which a perturbation approach captures the behavior of the system under consideration. These are the negative atomic detuning side of the frequency space where the photonic crystal density of states vanishes (I), the region surrounding the band-edge frequency (II) (which was inaccessible in the isotropic case), and the spectral region corresponding to moderate-large values of the atomic detuning (III). Remarkably, the non-Markovian effects associated with the fast variation of the density of states at the bandedge frequency (region II) can be analyzed within the framework of the Born approximation.

In the effective mass approximation and long-time limit, the memory kernel for a three-dimensional anisotropic photonic crystal is given by [34] (see also Appendix B 2)

$$
G_{A}\left(t-t^{\prime}\right)=-\beta_{A}^{1 / 2} \frac{e^{i\left[\pi / 4+\Delta_{A C}\left(t-t^{\prime}\right)\right]}}{\left(t-t^{\prime}\right)^{3 / 2}} .
$$

Here, $\Delta_{A C}=\omega_{A}-\omega_{C}$ is the detuning of the atomic frequency with respect to the band-edge frequency, and $\beta_{A}^{1 / 2}$ $=\omega_{A}^{2} d_{21}^{2} / 8 \hbar \epsilon_{0} \pi^{2} A^{3 / 2}$. An approximate form of the Laplace transform of anisotropic memory function is given in Appendix B 2:

$$
\widetilde{G}_{A}(p)=\beta_{A}^{1 / 2} e^{i \pi / 4} \sqrt{p-i \Delta_{A C}} .
$$

The temporal evolution of the excited atomic population is given in Appendix C. In Fig. 2, we plot the atomic popu- 


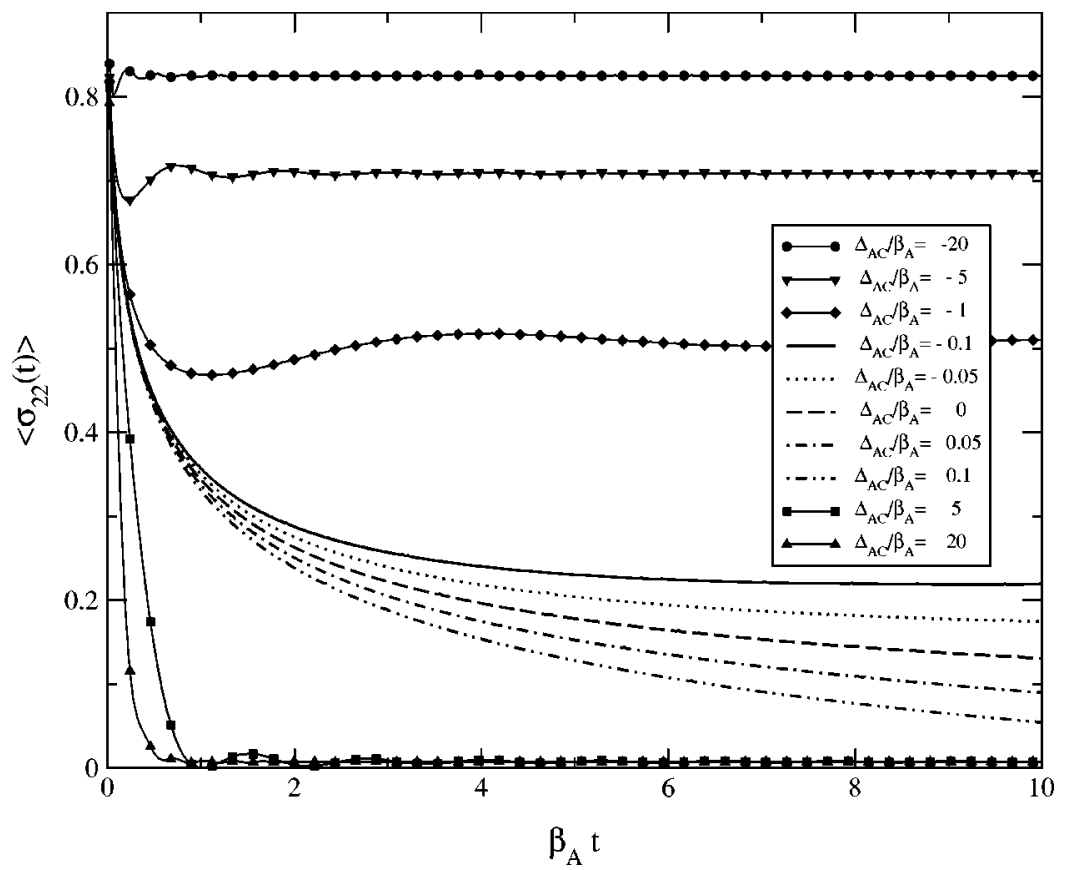

FIG. 2. Atomic population on the excited state, $\left\langle\sigma_{22}\right\rangle$, as a function of $\beta_{A} t$, for various values of the atomic detuning, from $\Delta_{A C} / \beta_{A}=$ -20 (circles) to $\Delta_{A C} / \beta_{A}=20$ (up triangles). The on-resonance case corresponds to the thick dashed curve. lation on the excited state as a function of the scaled time and for various values of the atomic detuning from the photonic band edge. It is now possible to realistically investigate the immediate neighborhood of the band-edge frequency. If the atomic frequency is detuned inside the gap, the atomic population again displays fractionalized inversion, for relatively small values of the atomic detuning. Even when the atomic frequency is detuned into the gap, a superposition of the continuum states leads to a bound state of the emitted photon to the atom.

As shown in Fig. 3, in the steady-state limit the dependence of atomic excited population on the atomic detuning is again very close to the one obtained using a nonperturbative approach [6].
Both methods show that, once the atomic frequency is detuned outside the gap, in the steady-state limit the atomic population vanishes. Outside the PBG, the remnant of the photon-atom bound state only becomes apparent when a small near-resonant driving field is applied to the system.

\section{Improved approximation scheme}

As stated in the Introduction, a critical issue for perturbation theory is the preservation of the positivity of the diagonal elements of the atomic density matrix operator. For certain initial conditions and for short-time scales, perturbationbased theories produce unphysical results. However, if one carefully restricts attention to the spectral regions over which

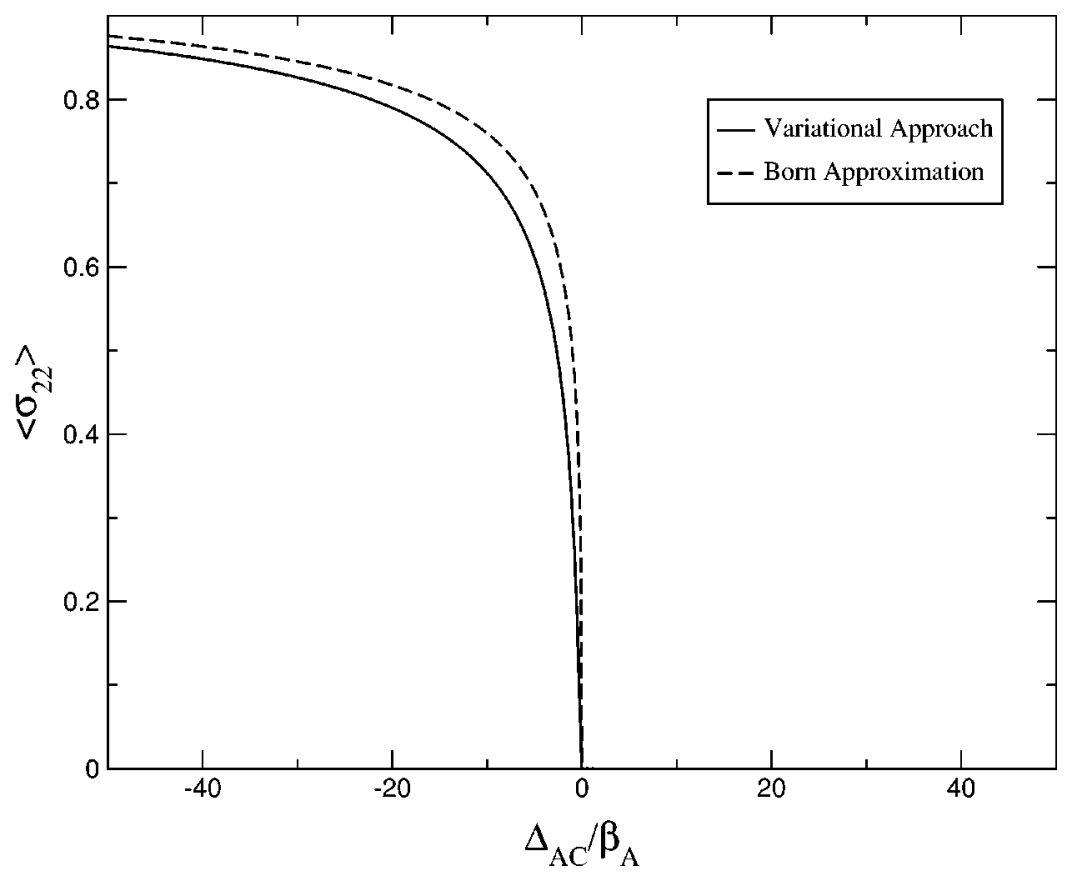

FIG. 3. Steady-state atomic population on the excited state, $\left\langle\sigma_{22}\right\rangle$, as a function of the atomic detuning $\Delta_{A C} / \beta_{A}$, within the Born approximation with leading non-Markovian corrections. 
a perturbation expansion is justified, it provides a very good approximation. As seen in the previous section, a consistent second-order perturbation theory allows one to investigate some of the non-Markovian features of the quantum optics in photonic crystals. It is has been pointed out that second-order perturbation theory gives correct results for single-operator expectation values [35]. The effects of neglecting the reservoir evolution appear only in expectation values of higherorder operator products. This observation suggests a simple approximation scheme which may be used to preserve the positivity of the excited atomic population. The scheme, however, is specific to the model under consideration. In our model, the average over the photonic reservoir degrees of freedom preserves some operatorial products. It can be shown easily that ${ }^{a} \sigma_{22} \equiv{ }^{a}\left(\sigma_{21} \sigma_{12}\right)=\left({ }^{a} \sigma_{21}\right)\left({ }^{a} \sigma_{12}\right)$ and ${ }^{a} \sigma_{11}$ $\equiv{ }^{a}\left(\sigma_{12} \sigma_{21}\right)=1-\left({ }^{a} \sigma_{21}\right)\left({ }^{a} \sigma_{12}\right) \neq\left({ }^{a} \sigma_{12}\right)\left({ }^{a} \sigma_{21}\right)$. Using these properties of the reduced atomic operators, we introduce an approximation scheme for the excited atomic population operator (once again we drop the $(a)$ superscript, which denotes the averaging over the reservoir degrees of freedom): $\sigma_{22}$ $=\sigma_{21} \sigma_{12}$ and $\sigma_{11}=1-\sigma_{22}$. Here, we substitute the approximants $\sigma_{21}^{(n)}=\sigma_{21}^{(0)}+\sum_{i=2}^{n} \delta \sigma_{21}^{(i)}$ and $\sigma_{12}^{(n)}=\sigma_{12}^{(0)}+\sum_{i=2}^{n} \delta \sigma_{12}^{(i)}$, which represent the $n$th perturbative solutions for $\sigma_{21}$ and $\sigma_{12}$, respectively, obtained by keeping terms up to $O\left(\Lambda^{n}\right)$ in the equations of motion.

Clearly, at any order $n$, this expansion for the reduced atomic operators preserves the trace of the Bloch vector $\vec{\sigma}$ $\left(\sigma_{11}+\sigma_{22}=1\right)$, ensures the positivity of the atomic populations [since $\sigma_{21}^{(n)}=\left(\sigma_{12}^{(n)}\right)^{\dagger}$ ], and converges to the exact solution as $n \rightarrow \infty$. The zeroth order corresponds to timeindependent atomic operators $\sigma_{21}^{(0)}(t)=\sigma_{21}(0), \quad \sigma_{22}^{(0)}(t)$ $=\sigma_{21}^{(0)}(0) \sigma_{12}^{(0)}(0)=\sigma_{22}(0)$, while, in the next order, the excited atomic population is given by $\sigma_{22}^{(2)}(t)=\sigma_{21}^{(2)}(t) \sigma_{12}^{(2)}(t)$. Here, $\sigma_{21,12}^{(2)}$ are the solutions of the equations of motion in which one keeps terms up to the second-order in $\Lambda$ (Born approximation), as in the previous section. The second-order expansion yields identical results (see Appendix C) with the variational Schrödinger equation approach described earlier [6].

\section{SINGLE ATOM DRIVEN BY AN EXTERNAL LASER FIELD}

\section{A. Model Hamiltonian}

We now consider a single stationary atom driven by an external single-mode laser field. We assume that the laser is in a coherent state $\left|\alpha \exp \left(-i \omega_{L} t\right)\right\rangle$, with $\alpha=|\alpha| e^{-i \phi_{L}}$, and that the occupation number of the laser mode $\bar{N}_{L}=\left|\alpha^{2}\right|$ is high enough such that one can disregard the influence of the atomic system on the laser-field radiation and average over the laser-field degrees of freedom. Under these assumptions, the interaction Hamiltonian $H_{A L}$ can be brought to the usual RWA form of the interaction between a two-level atom and a classical coherent monochromatic laser field:

$$
H_{A L}=\hbar \varepsilon\left(\sigma_{21} e^{-i\left(\omega_{L} t+\phi_{T}\right)}+\sigma_{12} e^{i\left(\omega_{L} t+\phi_{T}\right)}\right) .
$$

Here, $\varepsilon=\mathbf{d}_{21} \mathcal{E} / \hbar$ is the Rabi frequency, the laser electrical field magnitude $|\mathcal{E}|=\sqrt{\hbar \omega_{L} / 2 \epsilon_{0} \mathcal{V}} \sqrt{\bar{N}_{L}} e_{L}$, and all phase contributions are grouped in $\phi_{T}=\phi_{L}-\pi / 2$.

We now assume that the driven atomic system is coupled to the radiation reservoir of a photonic crystal. Using the notation introduced in Sec. I, the total Hamiltonian of the system is given by $H=H_{0}+H_{1}$, where

$$
\begin{aligned}
H_{0}= & \sum_{\lambda} \hbar \omega_{\lambda} a_{\lambda}^{\dagger} a_{\lambda}+\frac{1}{2} \hbar \omega_{A} \sigma_{3} \\
& +\hbar \varepsilon\left[\sigma_{21} e^{-i\left(\omega_{L} t+\phi_{T}\right)}+\sigma_{12} e^{i\left(\omega_{L} t+\phi_{T}\right)}\right], \\
& H_{1}=i \hbar \sum_{\lambda} g_{\lambda}\left(a_{\lambda}^{\dagger} \sigma_{12}-a_{\lambda} \sigma_{21}\right) .
\end{aligned}
$$

In order to eliminate the explicit time dependence of the Hamiltonian, we transform to a rotating frame of reference with the frequency $\omega_{L}$. In this frame of reference, the effective Hamiltonian [36] becomes

$$
\begin{gathered}
H^{\prime}=H_{0}^{\prime}+H_{1}^{\prime}, \\
H_{0}^{\prime}=\sum_{\lambda} \hbar \Delta_{\lambda} a_{\lambda}^{\dagger} a_{\lambda}+\frac{1}{2} \hbar \Delta_{A L} \sigma_{3}+\hbar \varepsilon\left(\sigma_{12}+\sigma_{21}\right), \\
H_{1}^{\prime}=i \hbar \sum_{\lambda} g_{\lambda}\left(a_{\lambda}^{\dagger} \sigma_{12}-a_{\lambda} \sigma_{21}\right),
\end{gathered}
$$

with $\Delta_{\lambda} \equiv \omega_{\lambda}-\omega_{L}$ and $\Delta_{A L} \equiv \omega_{A}-\omega_{L}$.

\section{B. Heisenberg equations of evolution}

\section{Heisenberg equations of evolution: Bare picture}

With the usual procedure of "adiabatic" elimination of the field variables, the Hamiltonian (4.3) generates the following Heisenberg equations of motion for the $\lambda$ mode of the radiation field, the atomic system polarization $\sigma_{21}$, and the atomic population inversion $\sigma_{3}$ :

$$
\begin{gathered}
a_{\lambda}(t)=\eta_{\lambda}(t)+\Lambda \int_{0}^{t} d t^{\prime} G_{\lambda}\left(t-t^{\prime}\right) \sigma_{12}\left(t^{\prime}\right) \\
\frac{d}{d t} \sigma_{21}(t)=\Lambda \eta^{\dagger}(t) \sigma_{3}(t)-i \varepsilon \sigma_{3}(t)+i \Delta_{A L} \sigma_{21}(t) \\
+\Lambda^{2} \int_{0}^{t} d t^{\prime} G\left(t-t^{\prime}\right) \sigma_{21}\left(t^{\prime}\right) \sigma_{3}(t) \\
\frac{d}{d t} \sigma_{3}(t)=-2 \Lambda \sigma_{21}(t) \eta(t)-2 i \varepsilon \sigma_{21}(t)-2 \Lambda^{2} \int_{0}^{t} d t^{\prime} \\
\times
\end{gathered}
$$

Since the noninteracting Hamiltonian contains the atomic operators $\sigma_{3}, \sigma_{12}, \sigma_{21}$ with different factors $\left(\Delta_{A L}, \varepsilon\right.$, respectively), $\sigma_{i j}(t) \approx e^{-i L_{0}\left(t-t^{\prime}\right)}\left\{\sigma_{i j}\left(t^{\prime}\right)\right\}$ cannot be decoupled in a simple form. The bare picture perturbation theory of the resonance fluorescence is tedious since the leading 
approximation of $\sigma_{i j}(t)$ contains combinations of all atomic operators [18]. Another possibility [16] is to displace one of the vacuum modes, such that it contains the external laser field. Then the corresponding noninteracting Hamiltonian becomes diagonal and the Born approximation may easily be applied. In this paper, we choose to diagonalize the noninteracting part of the Hamiltonian by transforming to the dressed atom basis. In the resulting Heisenberg picture, the operators no longer retain their atomic or electromagnetic character for times $t>0$. Nevertheless, the usual commutation relations are preserved during the temporal evolution.

\section{Heisenberg equations of evolution: Dressed picture}

The dressed atomic basis is defined as $|\widetilde{1}\rangle=c|1\rangle+s|2\rangle$, $|\widetilde{2}\rangle=-s|1\rangle+c|2\rangle$, where $c=\cos \phi, s=\sin \phi$, and $\sin ^{2} \phi$ $=\frac{1}{2}\left[1-\operatorname{sgn}\left(\Delta_{A L}\right) /\left(4 \epsilon^{2} / \Delta_{A L}^{2}+1\right)^{1 / 2}\right]$. The corresponding dressed atomic operators $R_{i j}=|\tilde{i}\rangle\langle\tilde{j}|(i, j=1,2), \quad R_{3}=R_{22}$ $-R_{11}$ are related to the bare atomic operators by the relations

$$
\begin{gathered}
\sigma_{12}=c s R_{3}+c^{2} R_{12}-s^{2} R_{21}, \\
\sigma_{21}=c s R_{3}-s^{2} R_{12}+c^{2} R_{21}, \\
\sigma_{3}=\left(c^{2}-s^{2}\right) R_{3}-2 c s\left(R_{12}+R_{21}\right) .
\end{gathered}
$$

This transformation leads to the noninteracting dressedstate Hamiltonian

$$
H_{0}=\hbar \Omega R_{3}+\hbar \sum_{\lambda} \Delta_{\lambda} a_{\lambda}^{\dagger} a_{\lambda}
$$

with $\Omega=\left[\epsilon^{2}+\Delta_{A L}^{2} / 4\right]^{1 / 2}$ the generalized Rabi frequency.

We define the time-dependent interaction picture Hamiltonian $\widetilde{H}_{1}=U^{\dagger}(t) H_{1} U(t)$, with the unitary transformation operator $U(t)=\exp \left(-i H_{0} t / \hbar\right)$. In this picture, the interaction Hamiltonian $\widetilde{H}_{1}$ takes the form

$$
\begin{aligned}
\tilde{H}_{1}= & i \hbar \Lambda \sum_{\lambda} g_{\lambda}\left[a _ { \lambda } ^ { \dagger } \left(\operatorname{cs} R_{3} e^{i \Delta_{\lambda} t}+c^{2} R_{12} e^{i\left(\Delta_{\lambda}-2 \Omega\right) t}\right.\right. \\
& \left.\left.-s^{2} R_{21} e^{i\left(\Delta_{\lambda}+2 \Omega\right) t}\right)\right]+ \text { H.c. }
\end{aligned}
$$

The dressed atomic operators in this interaction picture exhibit the time dependence given by $\widetilde{R}_{12}(t)$ $=R_{12}(0) \exp (-2 i \Omega t), \widetilde{R}_{21}(t)=R_{21}(0) \exp (2 i \Omega t)$, and $\widetilde{R}_{3}(t)$ $=R_{3}(0)$. Clearly, $\widetilde{R}_{3}(t), \widetilde{R}_{12}(t)$, and $\widetilde{R}_{21}(t)$ can be considered as source operators for the central component and left and right sidebands of the Mollow triplet at the frequencies $\omega_{L}, \omega_{L}-2 \Omega$, and $\omega_{L}+2 \Omega$. Hereafter, we drop the tilde on the interaction picture operators. The Hamiltonian given by Eq. (4.7) generates the following equations of motion:

$$
\begin{aligned}
\frac{d}{d t} a_{\lambda}(t)= & g_{\lambda} \Lambda\left\{c s R_{3} e^{i \Delta_{\lambda} t}+c^{2} R_{12} e^{i\left(\Delta_{\lambda}-2 \Omega\right) t}\right. \\
& \left.-s^{2} R_{21} e^{i\left(\Delta_{\lambda}+2 \Omega\right) t}\right\},
\end{aligned}
$$

$$
\begin{aligned}
\frac{d}{d t} R_{21}(t)= & \Lambda \sum_{\lambda} g_{\lambda}\left\{-2 c s a_{\lambda}^{\dagger} R_{21} e^{i \Delta_{\lambda} t}+c^{2} a_{\lambda}^{\dagger} R_{3} e^{i\left(\Delta_{\lambda}-2 \Omega\right) t}\right. \\
& \left.+2 c s R_{21} a_{\lambda} e^{-i \Delta_{\lambda} t}+s^{2} R_{3} a_{\lambda} e^{-i\left(\Delta_{\lambda}+2 \Omega\right) t}\right\} \\
\frac{d}{d t} R_{3}(t)= & -2 \Lambda \sum_{\lambda} g_{\lambda}\left\{s^{2} a_{\lambda}^{\dagger} R_{21} e^{i\left(\Delta_{\lambda}+2 \Omega\right) t}\right. \\
& \left.+c^{2} a_{\lambda}^{\dagger} R_{12} e^{i\left(\Delta_{\lambda}-2 \Omega\right) t}\right\}+ \text { H.c. }
\end{aligned}
$$

We adiabatically eliminate the field operators by formally integrating Eq. (4.8a) and substituting the result back into Eqs. (4.8b) and (4.8c). Further, we average over the field and atomic variables. We assume that the radiation field is initially in its vacuum state, i.e., $\left\langle a_{\lambda}(0)\right\rangle=\left\langle a_{\lambda}^{\dagger}(0)\right\rangle$ $=\left\langle a_{\lambda}(0) R_{i j}(t)\right\rangle=0$. In the Born approximation we retain terms up to the second order in $\Lambda$, and replace

$$
R_{i j}(t) \approx e^{-i L_{0}\left(t-t^{\prime}\right)}\left\{R_{i j}\left(t^{\prime}\right)\right\} \approx R_{i j}\left(t^{\prime}\right) .
$$

It follows that, in the Born approximation, $\left\langle R_{i j}(t) R_{m k}\left(t^{\prime}\right)\right\rangle$ $\approx\left\langle R_{i j}\left(t^{\prime}\right) R_{m k}\left(t^{\prime}\right)\right\rangle=\left\langle R_{i k}\left(t^{\prime}\right)\right\rangle \delta_{j m}$.

We introduce the memory functions $G_{0}\left(t-t^{\prime}\right)$ $=\Sigma_{\lambda} g_{\lambda}^{2} e^{-i \Delta_{\lambda}\left(t-t^{\prime}\right)}$ and $G_{ \pm}\left(t-t^{\prime}\right)=\Sigma_{\lambda} g_{\lambda}^{2} e^{-i\left(\Delta_{\lambda} \pm 2 \Omega\right)\left(t-t^{\prime}\right)}$. These functions characterize the memory effects of the central component, the right and left side bands of the Mollow spectrum, and describe the influence of the driving field on the atom-reservoir interaction (see Appendix D for the general equations of motion). The system of equations characterizing the evolution of the atomic operators is further simplified by making the secular approximation; i.e., the fast oscillating terms with frequencies $2 \Omega$ and $4 \Omega$ are discarded. This approximation is valid in the regime $\Omega>\beta$, where $\beta$ is the characteristic time scale of the evolution of the atomic system.

Under these assumptions, the temporal evolution of the atomic system is described by the following equations:

$$
\begin{aligned}
\frac{d}{d t}\left\langle R_{21}(t)\right\rangle= & -2 c^{2} s^{2} \int_{0}^{t} d t^{\prime} G_{0}^{*}\left(t-t^{\prime}\right)\left\langle R_{21}\left(t^{\prime}\right)\right\rangle \\
& -c^{4} \int_{0}^{t} d t^{\prime} G_{+}^{*}\left(t-t^{\prime}\right)\left\langle R_{21}\left(t^{\prime}\right)\right\rangle \\
& -2 c^{2} s^{2} \int_{0}^{t} d t^{\prime} G_{0}\left(t-t^{\prime}\right)\left\langle R_{21}\left(t^{\prime}\right)\right\rangle \\
& -s^{4} \int_{0}^{t} d t^{\prime} G_{-}\left(t-t^{\prime}\right)\left\langle R_{21}\left(t^{\prime}\right)\right\rangle, \quad(4.1 \\
\frac{d}{d t}\left\langle R_{3}(t)\right\rangle=- & 2 c^{4} \int_{0}^{t} d t^{\prime} G_{+}\left(t-t^{\prime}\right)\left\langle R_{22}\left(t^{\prime}\right)\right\rangle \\
+ & 2 s^{4} \int_{0}^{t} d t^{\prime} G_{-}\left(t-t^{\prime}\right)\left\langle R_{11}\left(t^{\prime}\right)\right\rangle+\text { H.c. }
\end{aligned}
$$




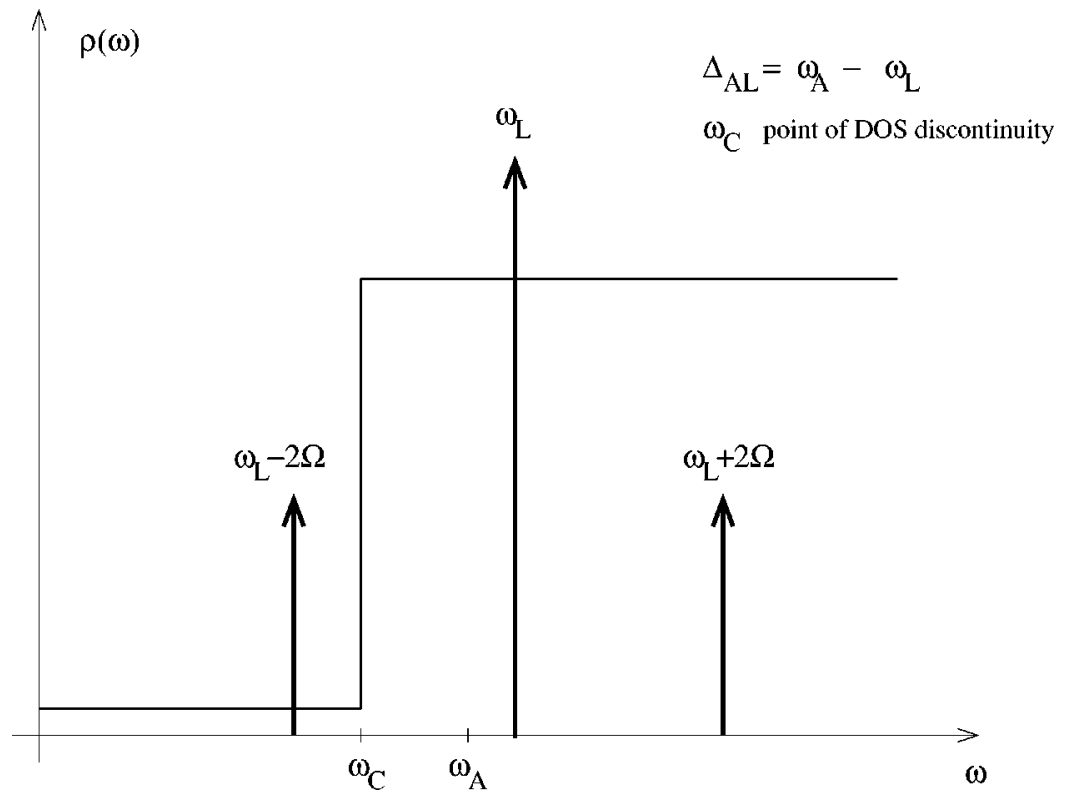

FIG. 4. Relevant frequencies and frequency scales in the case of a steplike density of states.

\section{Strong external laser field: The Markovian approximation}

In general, the memory functions $G_{0}\left(t-t^{\prime}\right)$ and $G_{+}(t$ $\left.-t^{\prime}\right)$ are determined by the radiation-field density of states. For a broadband, smoothly varying density of states of the reservoir (as in ordinary vacuum), the dependence of the memory functions on the external field can be ignored and $G_{0}\left(t-t^{\prime}\right)=G_{+}\left(t-t^{\prime}\right) \approx(\gamma / 2) \delta\left(t-t^{\prime}\right)$. However, the density of states of the photonic crystals exhibits band-edge and other Van Hove singularities, as described in the Introduction. In such a system with fast variations of the density of states in the spectral range given by $\left\{\omega_{L}-2 \Omega, \omega_{L}+2 \Omega\right\}$ (shown in Fig. 4), the distinctive memory functions introduced previously lead to qualitatively different behavior from an ordinary vacuum.

For a strong external laser field [7], the dressed frequencies $\omega_{L}, \omega_{L}-2 \Omega$, and $\omega_{L}+2 \Omega$ may be pushed far away from the band-edge singularity. In this section we assume that the photonic-mode density, while singular at one frequency, is smoothly varying over the spectral regions surrounding the dressed-state resonant frequencies $\omega_{L}, \omega_{L}$ $-2 \Omega$, and $\omega_{L}+2 \Omega$. The spectral components will experience very different densities of states and the memory functions in the Markov approximation are given by $G_{0}\left(t-t^{\prime}\right)$ $=\left(\gamma_{0} / 2\right) \delta\left(t-t^{\prime}\right), G_{+}\left(t-t^{\prime}\right)=\left(\gamma_{+} / 2\right) \delta\left(t-t^{\prime}\right)$, and $G_{-}(t$ $\left.-t^{\prime}\right)=\left(\gamma_{-} / 2\right) \delta\left(t-t^{\prime}\right)$. The spontaneous emission decay rates $\gamma_{0}=2 \pi \Sigma_{\lambda} g_{\lambda}^{2} \delta\left(\omega_{\lambda}-\omega_{L}\right), \quad \gamma_{-}=2 \pi \Sigma_{\lambda} g_{\lambda}^{2} \delta\left(\omega_{\lambda}-\omega_{L}\right.$ $+2 \Omega)$, and $\gamma_{+}=2 \pi \Sigma_{\lambda} g_{\lambda}^{2} \delta\left(\omega_{\lambda}-\omega_{L}-2 \Omega\right)$ are proportional to the density of modes at dressed-state resonant frequencies. The strong-field assumption ensures that the Mollow spectral components are well separated and the overlap between them is negligible (in fact this assumption requires a much stronger field than the one involved in the secular approximation).

Under these approximations, the temporal evolution of the dressed atomic polarization and inversion is then given by

$$
\left\langle R_{21}(t)\right\rangle=\left\langle R_{21}(0)\right\rangle \exp \left[-\frac{1}{2}\left(4 c^{2} s^{2} \gamma_{0}+c^{4} \gamma_{+}+s^{4} \gamma_{-}\right) t\right]
$$

$$
\begin{aligned}
\left\langle R_{3}(t)\right\rangle= & \left\langle R_{3}(0)\right\rangle \exp \left[-\left(c^{4} \gamma_{+}+s^{4} \gamma_{-}\right) t\right] \\
& -\frac{c^{4} \gamma_{+}-s^{4} \gamma_{-}}{c^{4} \gamma_{+}+s^{4} \gamma_{-}}\left\{1-\exp \left[-\left(c^{4} \gamma_{+}+s^{4} \gamma_{-}\right) t\right]\right\} .
\end{aligned}
$$

The bare operators are obtained by the transformation of Eq. (4.5), and the bare-state excited atomic population and polarization behavior is quite different from the one found in the ordinary vacuum case $[37,38]$ (these results correspond to setting $\gamma_{0}=\gamma_{-}=\gamma_{+}$).

In Figs. 5(a) and 5(b), we plot the bare excited atomic population as a function of the scaled time $\tau=\gamma_{+} t$ and the scaled resonance Rabi frequency $\epsilon /\left|\Delta_{A L}\right|$ for two values of the decay ratio $\gamma_{-} / \gamma_{+}$. The first figure corresponds to the ordinary vacuum case, with no singularity in the density of states $\left(\gamma_{+}=\gamma_{-}\right)$, and the second one to a confined photonic material, with an appreciable jump in its density of states $\gamma_{-} / \gamma_{+}=10^{-3}$. Both graphs share similar structures but there are some no table differences. For the confined photonic material, the time scale of the atomic evolution is given by the decay rate of the right side band of the Mollow spectrum $\left(\tau=\gamma_{+} t\right)$. For a given laser-field intensity and frequency, this time scale may be very different from the usual time scale $\left(\tau=\gamma_{0} t\right)$ encountered in the ordinary vacuum case. In an ordinary vacuum, the atomic system does not exhibit steady-state inversion. Consequently, conventional lasers require additional atomic levels to achieve atomic inversion. In the confined photonic material, for a given intensity of the laser field, the atomic system reaches positive inversion [Fig. 5(b)]. As shown in Fig. 6, if the jump in the photonic density of states is quite large (corresponding to small ratios $\left.\gamma_{-} / \gamma_{+}\right)$, the atomic system achieves nearly total inversion. 

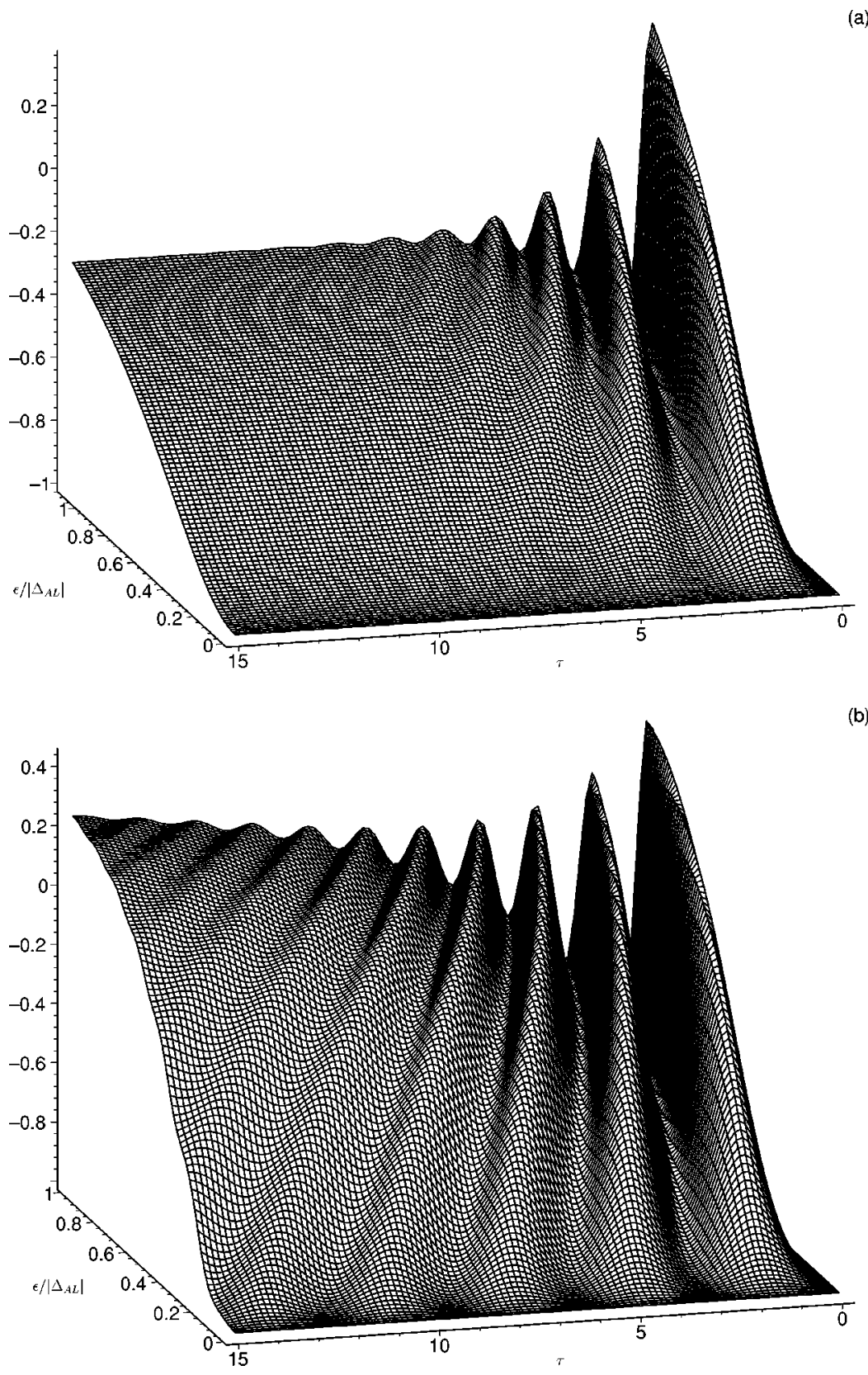

FIG. 5. (a) Atomic population inversion $\left\langle\sigma_{3}\right\rangle$ as a function of the scaled time $\tau=\gamma_{+} t$ and the intensity of the laser field $\epsilon /\left|\Delta_{A L}\right|$, for $\gamma_{-} / \gamma_{+}$ $=\gamma_{0} / \gamma_{+}=1, \quad \operatorname{sgn}\left(\Delta_{A L}\right)=-1, \quad \Omega / \gamma_{+}=2 . \quad$ (b) Atomic population inversion $\left\langle\sigma_{3}\right\rangle$ as a function of the scaled time $\tau=\gamma_{0} t$ and the intensity of the laser field $\epsilon /\left|\Delta_{A L}\right|$, for $\gamma_{0} / \gamma_{+}=1, \quad \gamma_{-} / \gamma_{+}$ $=10^{-3}, \operatorname{sgn}\left(\Delta_{A L}\right)=-1, \Omega / \gamma_{+}=2$.
This behavior is a consequence of the fact that the dressed state $|\tilde{1}\rangle$ (the left Mollow's sideband at the frequency $\omega_{L}$ $-2 \Omega$ ) is placed in the spectral region with a low density of states and with slow decay, whereas the dressed state $|\widetilde{2}\rangle$ (the right Mollow's sideband at the frequency $\omega_{L}+2 \Omega$ ) experiences a large density of states and a rapid decay. In the longtime limit, the population on the dressed state $|\tilde{1}\rangle$ is much larger than the atomic population in the dressed state $|\widetilde{2}\rangle$. This imbalance of the atomic population between the dressed states is responsible for the atomic inversion in the bare picture.

However, even though the single-atom system reaches positive inversion, the transition from negative to positive inversion is a smooth and gradual one in this Markov picture; i.e., there is no sharp switching effect as a function of applied field.

\section{Switching by a moderate external field: Non-Markovian case}

For weak and moderate external fields, Mollow's spectral components may remain close to the density of states discontinuity and it is necessary to solve the Eqs. (4.10a) and (4.10b) without recourse to the Markov approximation. For this purpose, we introduce Laplace transforms of the atomic variables, $\quad x(p)=\widetilde{R}_{3}(p)=\mathcal{L}\left\{\left\langle R_{3}(t)\right\rangle\right\} \quad$ and $\quad z(p)=\widetilde{R}_{21}(p)$ $=\mathcal{L}\left\{\left\langle R_{21}(t)\right\rangle\right\}$ and the memory functions $\widetilde{G}_{0}(p)$ $=\mathcal{L}\left\{G_{0}(t)\right\}, \quad \widetilde{G}_{+}(p)=\mathcal{L}\left\{G_{+}(t)\right\}$, and $\widetilde{G}_{-}(p)=\mathcal{L}\left\{G_{-}(t)\right\}$, where $\widetilde{f}(p)=\mathcal{L}\{f(t)\}=\int_{0}^{\infty} e^{-p t} f(t)$. With this notation, the solution of the evolution equations is given by

$$
x(p)=\frac{x_{0} p+\left[s^{4} \widetilde{G}_{-}(p)-c^{4} \widetilde{G}_{+}(p)+\text { c.c. }\right]}{p\left\{p+\left[c^{4} \widetilde{G}_{+}(p)+s^{4} \widetilde{G}_{-}(p)+\text { c.c. }\right]\right\}}
$$




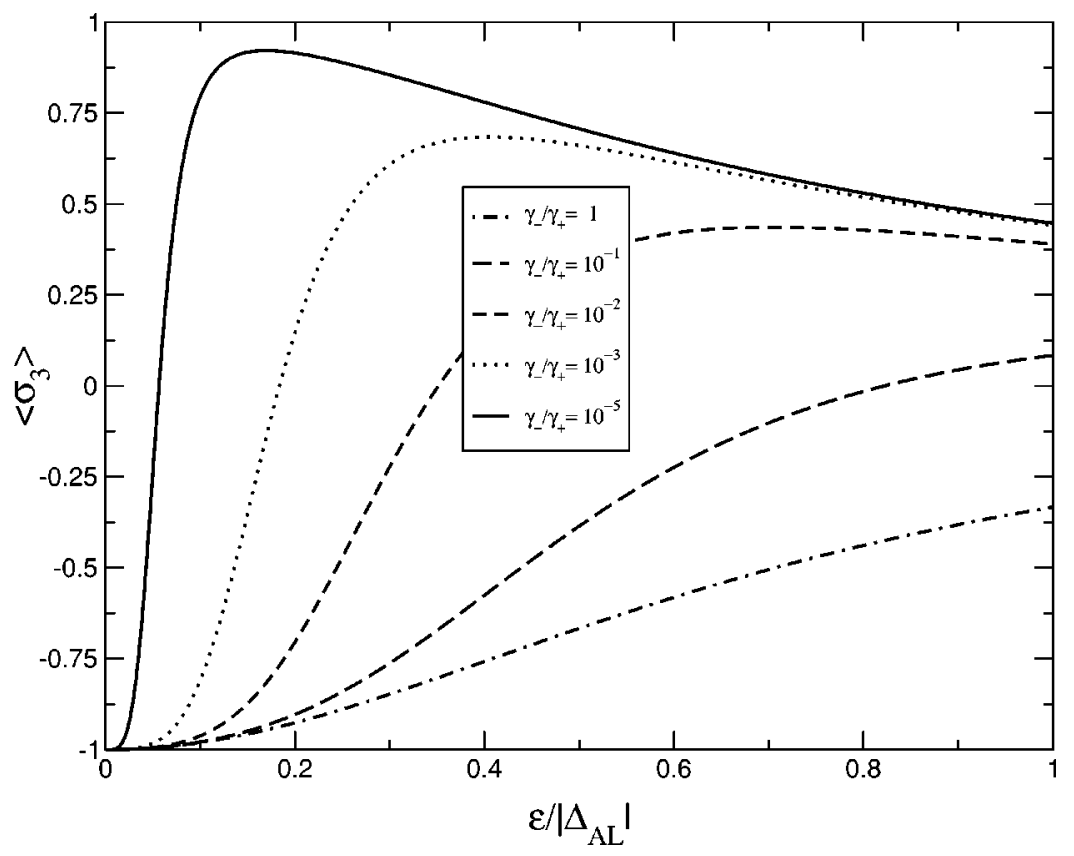

FIG. 6. Steady-state atomic population inversion $\left\langle\sigma_{3}\right\rangle$ as a function of $\epsilon /\left|\Delta_{A L}\right|$, for various discontinuities in the density of states, $\gamma_{-} / \gamma_{+}$ $=1$ (dot-dashed curve), $\gamma_{-} / \gamma_{+}=10^{-1}$ (longdashed curve), $\quad \gamma_{-} / \gamma_{+}=10^{-2}$ (short-dashed curve), and $\gamma_{+} / \gamma_{+}=10^{-3}$ (thin solid curve), $\gamma_{-} / \gamma_{+}=10^{-5}$ (thick solid curve). The atomic detuning is negative, $\operatorname{sgn}\left(\Delta_{A L}\right)=-1$.

$$
z(p)=\frac{z_{0}}{p+c^{4} \widetilde{G}_{+}^{*}(p)+s^{4} \widetilde{G}_{-}(p)+2 c^{2} s^{2}\left[\widetilde{G}_{0}(p)+\widetilde{G}_{0}^{*}(p)\right]},
$$

where $x_{0}=\left\langle R_{3}(0)\right\rangle$ and $z_{0}=\left\langle R_{21}(0)\right\rangle$ are the initial values of the excited atomic population and polarization, respectively. These are determined by the initial values of the barestate inversion and polarization through Eqs. (4.5). The time evolution of the dressed state atomic variables is given by $\left\langle R_{3}(t)\right\rangle=\mathcal{L}^{-1}\left\{\widetilde{R}_{3}(p)\right\}=\mathcal{L}^{-1}\{x(p)\}$, and their bare-state counterparts are obtained using Eq. (4.5). We will use these results to analyze the temporal and the steady-state behavior of the atomic population and polarization of a two-level atom placed in a 3D anisotropic photonic crystal, with the relevant frequencies shown in Fig. 7.

The memory function and the corresponding Laplace transform for a three-dimensional photonic crystal have been evaluated [6], and in the long-time limit they are given by Eqs. (3.11) and (3.12). The Laplace transforms of the memory functions for the anisotropic photonic band edge are

$$
\begin{gathered}
\widetilde{G}_{0}(p)=\mathcal{L}\left\{G_{0}(t)\right\}=\sqrt{i} \beta_{A}^{1 / 2} \sqrt{p_{0}}, \\
\widetilde{G}_{ \pm}(p)=\mathcal{L}\left\{G_{ \pm}(t)\right\}=\sqrt{i} \beta_{A}^{1 / 2} \sqrt{p_{\mp}},
\end{gathered}
$$

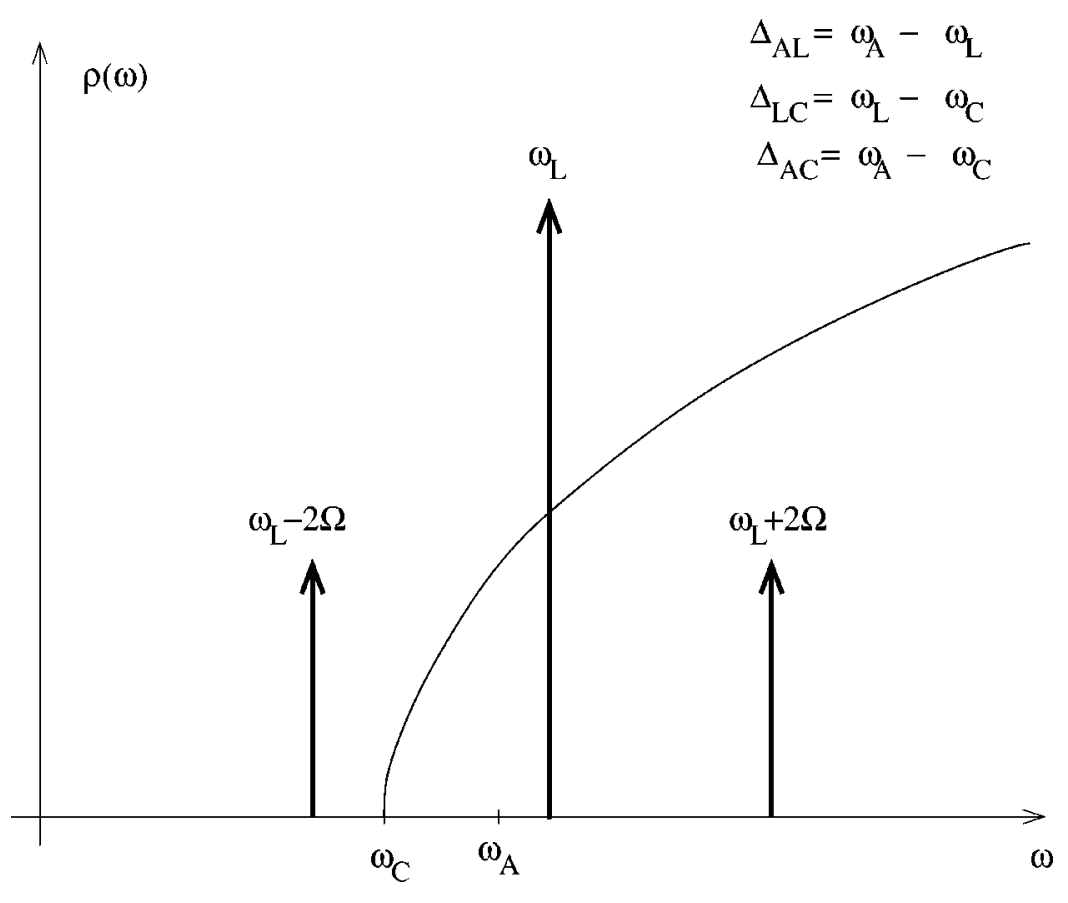

FIG. 7. Relevant frequencies and frequency scales in the case of an anisotropic density of states. 


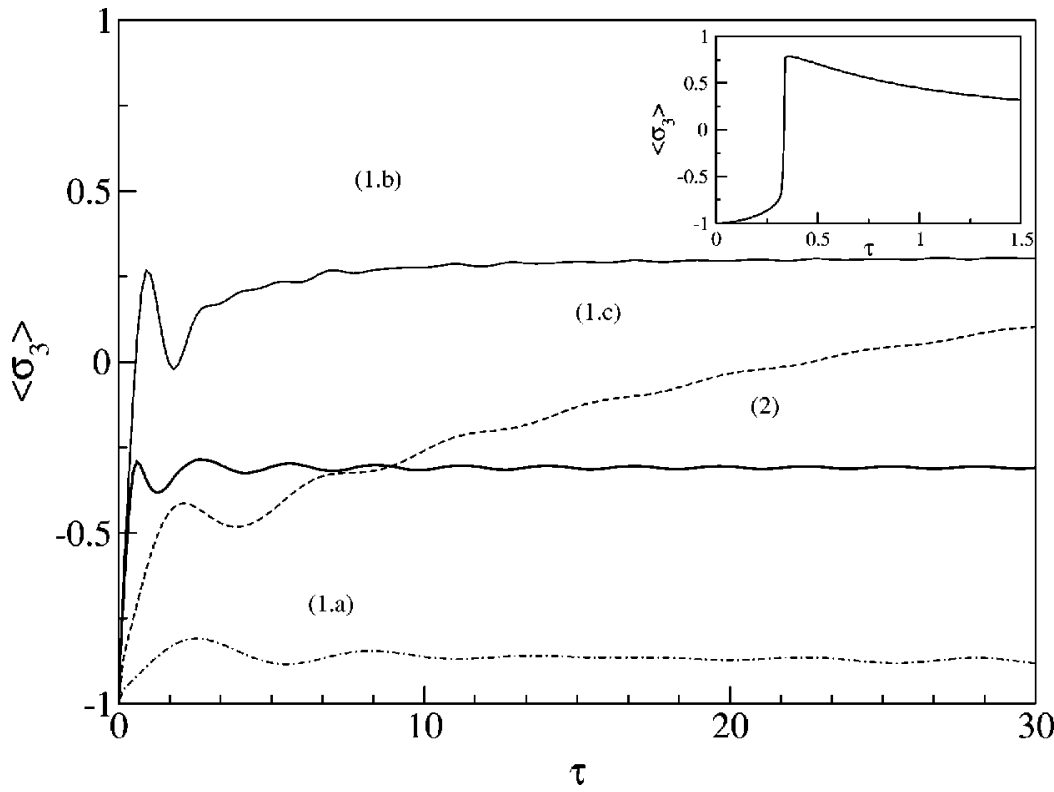

FIG. 8. Atomic population inversion $\left\langle\sigma_{3}(t)\right\rangle$ as a function of the scaled time $\tau=\beta_{A} t$, for various values of the laser intensity (curves $1 . \mathrm{a}, 1 . \mathrm{b}$, 1.c): $\epsilon / \beta_{A}=0.25$ (1.a), $\epsilon / \beta_{A}=0.5$ (1.b), and $\epsilon / \beta_{A}=1.25$ (1.c). The laser field frequency detuning is $\Delta_{L C} / \beta_{A} \equiv\left(\omega_{L}-\omega_{C}\right) / \beta_{A}=1.2$. Curve 2 corresponds to $\epsilon / \beta_{A}=1$ and $\Delta_{L C} / \beta_{A}=20$. The inset shows the steady-state atomic population inversion $\left\langle\sigma_{3}\right\rangle$ as a function of $\epsilon / \beta_{A}$, when the laser-field frequency detuning is $\Delta_{L C} / \beta_{A}=1.2$. The atomic detuning for all curves is $\Delta_{A L}=$ $-\beta_{A}$.

where $p_{0} \equiv p-i \Delta_{L C}, \quad p_{\mp} \equiv p-i \Delta_{L C} \pm 2 i \Omega$, and $\Delta_{L C} \equiv \omega_{L}$ $-\omega_{C}$.

In the Laplace space, the evolution of the dressed atomic population and polarization satisfies Eqs. (4.12):

$$
x(p)=\frac{x_{0} p / \beta_{A}^{1 / 2}+2 s^{4} \operatorname{Re}\left[\sqrt{i} \sqrt{p_{+}}\right]-2 c^{4} \operatorname{Re}\left[\sqrt{i} \sqrt{p_{-}}\right]}{p\left(p / \beta_{A}^{1 / 2}+2 c^{4} \operatorname{Re}\left[\sqrt{i} \sqrt{p_{-}}\right]+2 s^{4} \operatorname{Re}\left[\sqrt{i} \sqrt{p_{+}}\right]\right)},
$$

$z(p)=\frac{z_{0} \beta_{A}^{1 / 2}}{p / \beta_{A}^{1 / 2}+c^{4} \sqrt{-i} \sqrt{p_{-}}+s^{4} \sqrt{i} \sqrt{p_{+}}+4 c^{2} s^{2} \operatorname{Re}\left[\sqrt{i} \sqrt{p_{0}}\right]}$.

The time-dependent dressed atomic variables are given by the inverse Laplace transformation $\left\langle R_{3}(t)\right\rangle=\mathcal{L}^{-1}\{x(p)\}$, $\left\langle R_{21}(t)\right\rangle=\mathcal{L}^{-1}\{z(p)\}$, where

$$
f(t)=\mathcal{L}^{-1}\{\widetilde{f}(p)\}=\frac{1}{2 \pi i} \int_{\varepsilon-i \infty}^{\varepsilon+i \infty} d p e^{p t} \widetilde{f}(p) .
$$

Here, the real number $\varepsilon$ is chosen so that $p=\varepsilon$ lies to the right of all singularities (poles and branch points) of the function to be integrated. The system (4.12) is simplified by scaling the time variable by $\beta_{A}^{1 / 2}$ (the frequency is then scaled with $1 / \beta_{A}^{1 / 2}$ ). We numerically evaluate the inverse Laplace transforms of the atomic variables using an Adams algorithm [39]. Once the Laplace inversion is performed, the bare-state atomic averages are obtained through Eq. (4.5).

In Fig. 8, we plot the excited atomic population inversion for some specific choices of the system parameters. If the left Mollow sideband is driven from outside the gap, $\Delta_{L C} \leqslant 2 \Omega$ [curve $1(\mathrm{a})$ ], to inside the gap, $\Delta_{L C}>2 \Omega$ [curves 1(b) and $1(\mathrm{c})]$, the atomic system becomes inverted in the stationary state [1(b) and 1(c)] [the actual stationary values, for $\tau$ $=t \beta_{A}>1000$, of the atomic inversion are $\left\langle\sigma_{3}\right\rangle^{s t}=$ -0.864694 for $1(\mathrm{a}),\left\langle\sigma_{3}\right\rangle^{s t}=0.370638$ for $1(\mathrm{~b})$, and $\left\langle\sigma_{3}\right\rangle^{s t}$ $=0.706475$ for $1(\mathrm{c})]$. The transition described above can be accomplished by starting with a given parametric configuration in which the left component is placed outside the gap near the band-edge frequency, and the intensity of the external laser field is gradually increased. Far outside the gap ( $\Delta_{L C} \gg 2 \Omega$ for curve 2 ), the influence of the band-edge singularity becomes negligible, and the system reaches the ordinary vacuum case regime. The inset shows the steady-state inversion as a function of applied-field intensity for a specific set of detuning frequencies. The analytical results are given in the next section.

\section{E. Steady-state solution}

The derivation of a steady-state solution for the atomic inversion is facilitated by the identity $\lim _{t \rightarrow \infty}\{F(t)\}$ $=\lim _{p \rightarrow 0}\{p \widetilde{f}(p)\}[40-42]$, where $\widetilde{f}=\mathcal{L}\{F\}$ is the Laplace transform of $F$. Since $z(p)$ and $x(p)$ have only complex (not purely imaginary poles), it follows that the steady state dressed atomic polarization vanishes: $\left\langle R_{21}\right\rangle^{s t}$ $=\lim _{t \rightarrow \infty}\left\langle R_{21}(t)\right\rangle=0$. However, the dressed excited atomic population, $\left\langle R_{3}\right\rangle^{s t}=\lim _{t \rightarrow \infty}\left\langle R_{3}(t)\right\rangle$ has a nontrivial behavior:

$$
\begin{aligned}
& \left\langle R_{3}\right\rangle^{s t} \\
& \quad= \begin{cases}-1 & \text { if } \Delta_{L C} \leqslant 2 \Omega, \\
\frac{s^{4}-c^{4} \sqrt{\left(\Delta_{L C}+2 \Omega\right) /\left(\Delta_{L C}-2 \Omega\right)}}{s^{4}+c^{4} \sqrt{\left(\Delta_{L C}+2 \Omega\right) /\left(\Delta_{L C}-2 \Omega\right)}} & \text { if } \Delta_{L C}>2 \Omega .\end{cases}
\end{aligned}
$$

We check the consistency of the solution in two limiting cases. First, if we place the Mollow spectral components far outside the gap $\Delta_{L C} \gg 2 \Omega$, the system reaches the ordinary vacuum behavior:

$$
\left\langle R_{3}\right\rangle^{s t}=-\frac{c^{4}-s^{4}}{c^{4}+s^{4}} \Rightarrow\left\langle\sigma_{22}\right\rangle^{s t}=-\frac{\left(c^{2}-s^{2}\right)^{2}}{c^{4}+s^{4}} .
$$


This limiting case is consistent with the Markovian approach (far away from the density of states singularity, the photonic crystal reservoir of modes is a Markovian reservoir). Accordingly, Eq. (4.16) can also be obtained in the Markovian formalism developed in Sec. IV C, by substituting $\gamma_{+}=\gamma_{-}$ $=\gamma_{0}$ in Eqs. (4.11a) and (4.11b). On the other hand, if we place the left spectral component inside the gap $\left(\Delta_{L C}\right.$ $\ll 2 \Omega$ ), the atomic system becomes trapped in the $|\widetilde{1}\rangle$ state:

$$
\left\langle R_{3}\right\rangle^{s t}=-1 \Rightarrow\left\langle\sigma_{22}\right\rangle^{s t}=s^{2}-c^{2} .
$$

Consider a near-resonant laser excitation $\left(\omega_{L} \approx \omega_{A}\right)$. If we begin with an atom with resonant transition frequency $\omega_{A}$ just outside the photonic band gap (say, for instance, $\Delta_{A C}$ $\equiv \omega_{A}-\omega_{C}>0$ ), it is possible to drive the system through the transition described above, simply by increasing the applied field intensity. For a nearly resonant laser excitation $\left(\omega_{L}\right.$ $\approx \omega_{A}$ ), the left Mollow sideband (at frequency $\omega_{A}-2 \Omega$ ) passes through the photonic band-edge frequency when $\Omega_{c r i t}=\Delta_{L C} / 2$, while the other Mollow spectral components remain outside the gap. At this critical laser intensity, the atomic population exhibits switching from a noninverted state to an inverted state. In the bare picture, the atomic inversion can be expressed as

$$
\left\langle\sigma_{22}\right\rangle^{s t}=\left\{\begin{array}{lll}
s^{2}-c^{2} & \text { if } \Delta_{L C} \leqslant 2 \Omega, \\
\left(c^{2}-s^{2}\right) \frac{s^{4}-c^{4} \sqrt{\frac{\Delta_{L C}+2 \Omega}{\Delta_{L C}-2 \Omega}}}{s^{4}+c^{4} \sqrt{\frac{\Delta_{L C}+2 \Omega}{\Delta_{L C}-2 \Omega}}} & \text { if } \quad \Delta_{L C}>2 \Omega .
\end{array}\right.
$$

It is apparent from Fig. 9(a) that, for moderate values of the laser intensity, the atomic system switches very sharply from the ground state to the excited state, at a critical value of $\epsilon$. This switching behavior is caused by the very sensitive dependence of the dressed atomic population on the relative position of the Mollow spectrum components. The magnitude of this effect depends on the actual value of the laser detuning with respect to the band-edge frequency, $\Delta_{L C}$ $=\omega_{L}-\omega_{C}$. This interplay between the control parameters is shown in Fig. 9(b), where now the atomic population dis- plays sharp switching behavior as a function of the detuning of the laser-field frequency, for various choices of the applied laser-field intensity.

A similar switching behavior was found in Ref. [7], in the context of collective behavior of an ensemble of two-level atoms placed in a confined photonic material and driven by an external laser field. As the left sideband of the Mollow spectrum was placed inside the gap of a photonic crystal or other low-DOS region, while the other spectral components were placed in a high-DOS region, the atomic population on the excited state showed a sharp collective jump. The switching behavior described in [7] is a collective effect (for a single-atom case, sharp switching is absent), strongly dependent on the atomic density. The Markov approximation required the use of a strong external laser field which drives the Mollow spectral components away from the photonic density of states singularity, so that, over the width of each component, the density of states is smooth $\left(\Omega \gg N \gamma_{0}, \Omega\right.$ $\gg N \gamma_{ \pm}$, where $\gamma_{0}, \gamma_{ \pm}$represent the decay rates of the central, right and left components of the Mollow triplet and $N$ is the number of atoms). In the present non-Markovian case, we describe single-atom switching, which occurs for moderate values of the laser intensity, due to the fast variation of the density of states near the band-edge frequency, the spectral range carefully avoided in Ref. [7].

\section{F. Influence of dephasing interactions and nonradiative relaxation}

In order to make closer contact with experiment, we include phenomenological decay rates $1 / T_{1}^{n r}$ and $1 / T_{2}^{n r}$ associated with other (nonradiative) decay and dephasing, respectively. Deep inside the gap, where radiative decay is negligible, the nonradiative contribution may become very important. In this case, $T_{1}^{n r}$ and $T_{2}^{n r}$ may be considered as empirical constants. The nonradiative decay may come from phonon-assisted transitions if the atom is placed in a solid matrix. Dephasing occurs if an atomic vapor is placed in the photonic crystal voids and is collisionally perturbed by the other atoms. If the atom is implanted in the dielectric region, the interaction with lattice vibrations of the host dielectric material (elastic scattering of the phonons on the atomic system) will cause dephasing.

The effect of these additional decay and dephasing mechanisms is investigated in Appendix D. Here, we present only the steady-state results for the dressed atomic population inversion and polarization:

$$
\begin{aligned}
& \left\langle R_{3}\right\rangle^{s t} \\
& \quad=\left\{\begin{array}{l}
{\left[-2 c^{4} \sqrt{\Delta_{L C}+2 \Omega}+\left(s^{2}-c^{2}\right) / \widetilde{T}_{1}^{n r}\right]\left[2 c^{4} \sqrt{\Delta_{L C}+2 \Omega}+4 s^{2} c^{2} / \widetilde{T}_{2}^{n r}+\left(s^{2}-c^{2}\right)^{2} / \widetilde{T}_{1}^{n r}\right]^{-1} \text { if } \Delta_{L C} \leqslant 2 \Omega,} \\
{\left[2 s^{4} \sqrt{\Delta_{L C}-2 \Omega}-2 c^{4} \sqrt{\Delta_{L C}+2 \Omega}+\left(s^{2}-c^{2}\right) / \widetilde{T}_{1}^{n r}\right)\left[2 s^{4} \sqrt{\Delta_{L C}-2 \Omega}+2 c^{4} \sqrt{\Delta_{L C}+2 \Omega}+4 s^{2} c^{2} / \widetilde{T}_{2}^{n r}+\left(s^{2}-c^{2}\right)^{2} / \widetilde{T}_{1}^{n r}\right]^{-1}} \\
\text { if } \Delta_{L C}>2 \Omega,
\end{array}\right.
\end{aligned}
$$



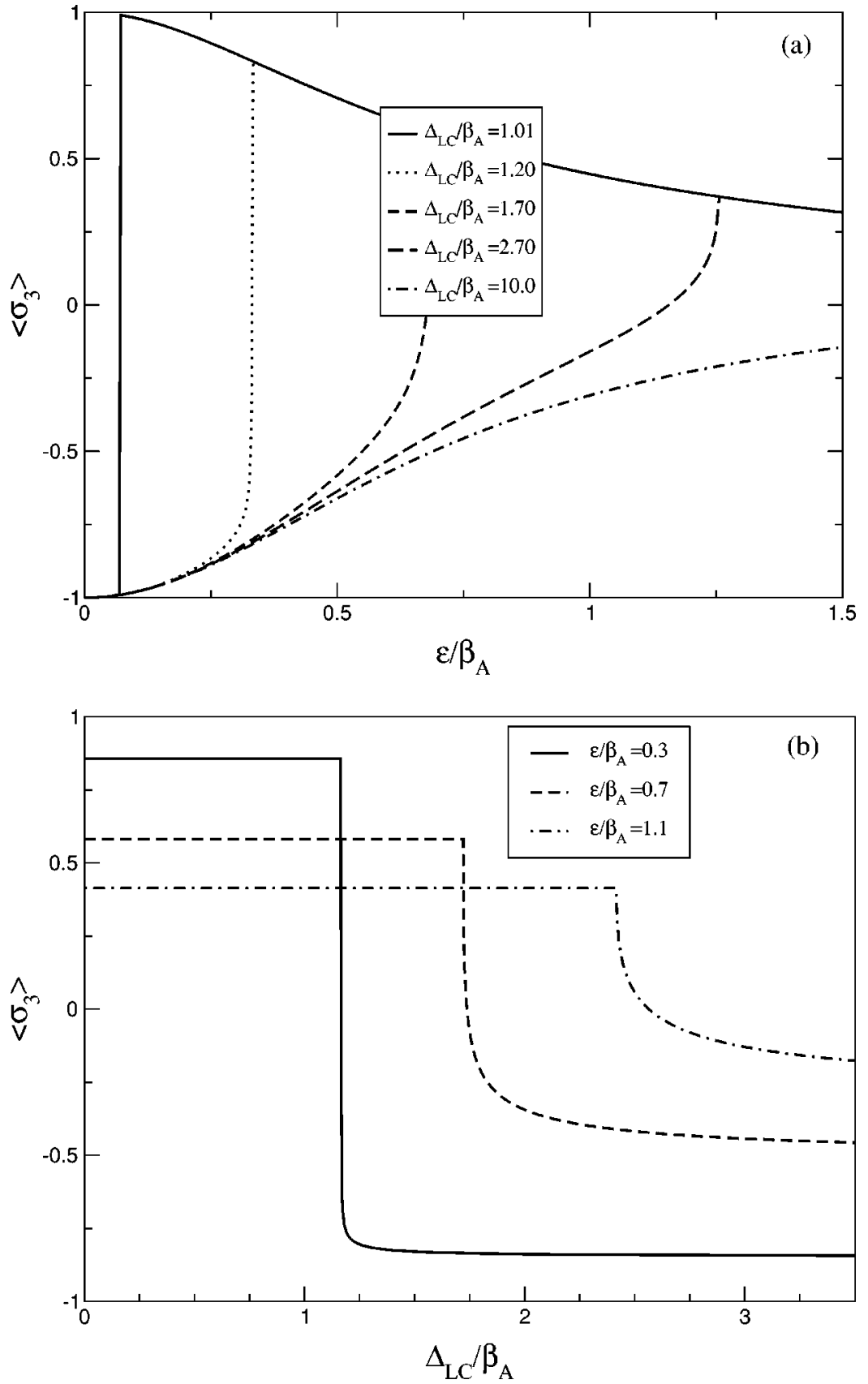

FIG. 9. (a) Atomic population inversion $\left\langle\sigma_{3}\right\rangle$ as a function of the laser intensity $\epsilon / \beta_{A}$, for different values of the laser detuning: $\Delta_{L C} / \beta_{A}$ $=1.01$ (solid curve), $\Delta_{L C} / \beta_{A}=1.20$ (dotted curve), $\Delta_{L C} / \beta_{A}=1.70$ (dashed curve), $\Delta_{L C} / \beta_{A}$ $=2.70$ (long-dashed curve), and $\Delta_{L C} / \beta_{A}=10.0$ (dot-dashed curve). The atomic detuning is $\Delta_{A L}$ $=-\beta_{A}$. (b) Atomic inversion $\left\langle\sigma_{3}\right\rangle$ as a function of the laser detuning $\Delta_{L C} / \beta_{A}$, for different values of the laser intensity: $\epsilon / \beta_{A}=0.3$ (solid curve), and $\epsilon / \beta_{A}=0.7$ (dotted curve), $\epsilon / \beta_{A}=1.1$ (dashed curve). The atomic detuning is $\Delta_{A L}=$ $-\beta_{A}$. where we introduced the scaled nonradiative decay and dephasing times $\widetilde{T}_{1}^{n r}=T_{1}^{n r} \beta_{A}^{1 / 2}$, and $\widetilde{T}_{2}^{n r}=T_{2}^{n r} \beta_{A}^{1 / 2}$, respectively.

Clearly, the additional decay and dephasing mechanisms tend to weaken the switching effect. The robustness of the switching effect follows from an estimate of the time scale factor in photonic band gap materials. In the case of the isotropic model, the time scale factor $\beta_{I}$ can be expressed as

$$
\beta_{I}=\omega_{C} \frac{1}{{\widetilde{A_{I}}}^{1 / 3}} 3 \sqrt{\frac{1}{16 \pi}\left(\frac{\gamma}{\omega_{A}}\right)^{2}\left(\frac{c k_{0}}{\omega_{C}}\right)^{4}},
$$

where we introduced the dimensionless constant $\widetilde{A_{I}}$ $=A_{I} /\left(c^{2} / \omega_{C}\right)$. The values of $\omega_{C}, k_{0}$, and $\widetilde{A_{I}}$, are determined by the specifics of the dielectric structure considered. However, the isotropic model is obtained by generalizing the dispersion relation of a one-dimensional Bragg stack to all $\mathbf{k}$ space directions [6], and, implicitly, the dependence of the time factor scale on the curvature of the dispersion relation $\left(\widetilde{A_{I}}\right)$ is reduced. In the optical domain, an estimate of $\beta_{I}$ produces $0.8 \gamma \leqslant \beta_{I} \leqslant 10 \gamma$.

The situation is quite different for the anisotropic model. In this case, the time scale factor $\beta_{A}$ can be expressed as

$$
\beta_{A}=\omega_{C} \frac{1}{{\overline{A_{A}}}^{3}} \frac{9}{256 \pi}\left(\frac{\gamma}{\omega_{21}}\right)^{2},
$$

and we obtain a much stronger dependence on the curvature of the dispersion relation. Moreover, real band structure cal- 


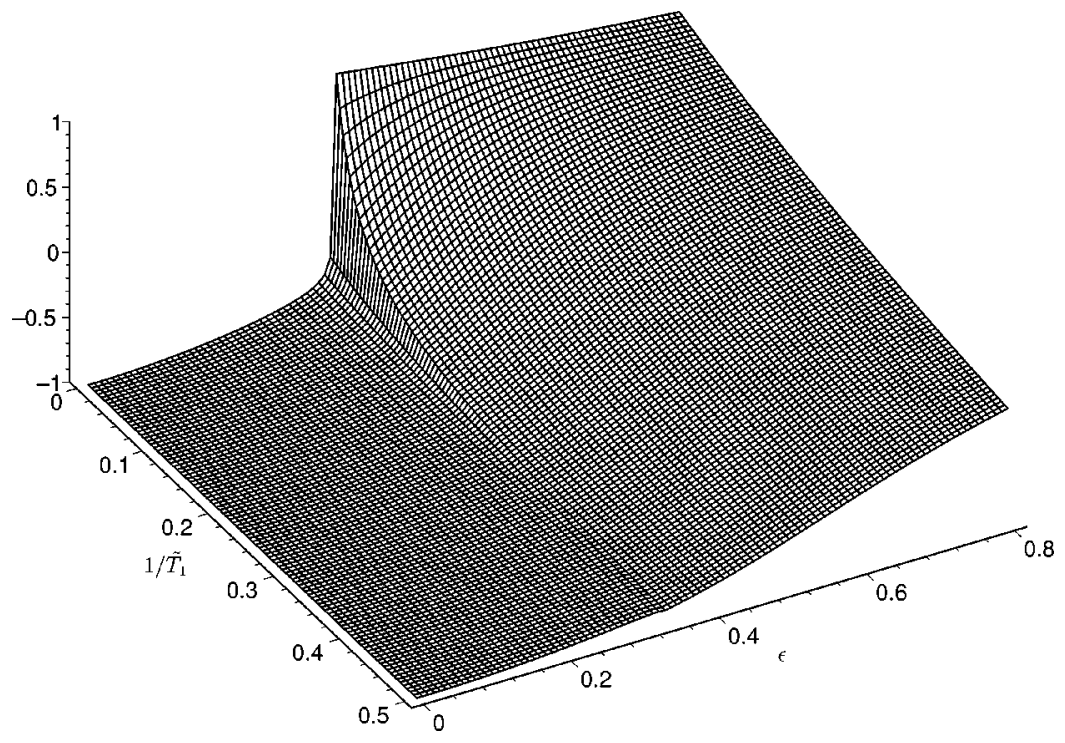

FIG. 10. Atomic population inversion $\left\langle\sigma_{3}\right\rangle$, as a function of $\epsilon / \beta_{A}$, and the nonradiative decay rate $1 / \widetilde{T}_{1}^{n r}$. The laser-field frequency detuning is $\Delta_{L C} / \beta_{A}=1.2, \Delta_{A L} / \beta_{A}=-1$, and $\Delta_{A C} / \beta_{A}=0.2$.

culations show an extremely sensitive dependence of the curvature of the dispersion relation on the specific direction in the reciprocal space $\left(\mathbf{k}_{0}\right)$ and produce a wide range of values for the dimensionless parameter $\widetilde{A_{A}}$.

We are using in our numerical estimations a time scale factor range $0.8 \gamma \leqslant \beta_{A} \leqslant 10 \gamma$, but point out that a more accurate estimation has to be obtained by using band structure calculations for a real photonic crystal (along the lines given in Appendix A).

Clearly, as shown in Fig. 10 and Fig. 11, a sizable switching effect is present even when $1 / \widetilde{T}_{1}^{n r}, 1 / \widetilde{T}_{2}^{n r} \approx \beta_{A}$. It is also apparent that the nonradiative decay contribution (Fig. 10) is much more deleterious to switching effects than the dephasing mechanisms (Fig. 11).

\section{CONCLUSIONS}

In this paper, we analyzed the leading non-Markovian corrections to resonance fluorescence in photonic band-gap materials. By means of a perturbative expansion of the
Heisenberg equations of motion in terms of the coupling strength of the atomic system to the radiation reservoir of modes, we recaptured the most important non-Markovian effects caused by the strong variation of the photonic density of states with frequency. In the context of spontaneous emission, our approach recaptures the physics of photon-atom bound state.

Our results provide a clear distinction between the Markov approximation and the Born approximation in the context of photonic band-gap materials. The Markov approximation is related to memory effects of the photonic radiation reservoir, whereas the Born approximation is associated with the strength of the coupling between the atom and the reservoir. In the case of realistic photonic crystals models, the essential non-Markovian effect leading to the photon-atom bound state can be captured even within the Born approximation.

We predict that a single atom in a PBG material driven by a external laser field with a moderate intensity will exhibit switching behavior as a function of both laser-field intensity

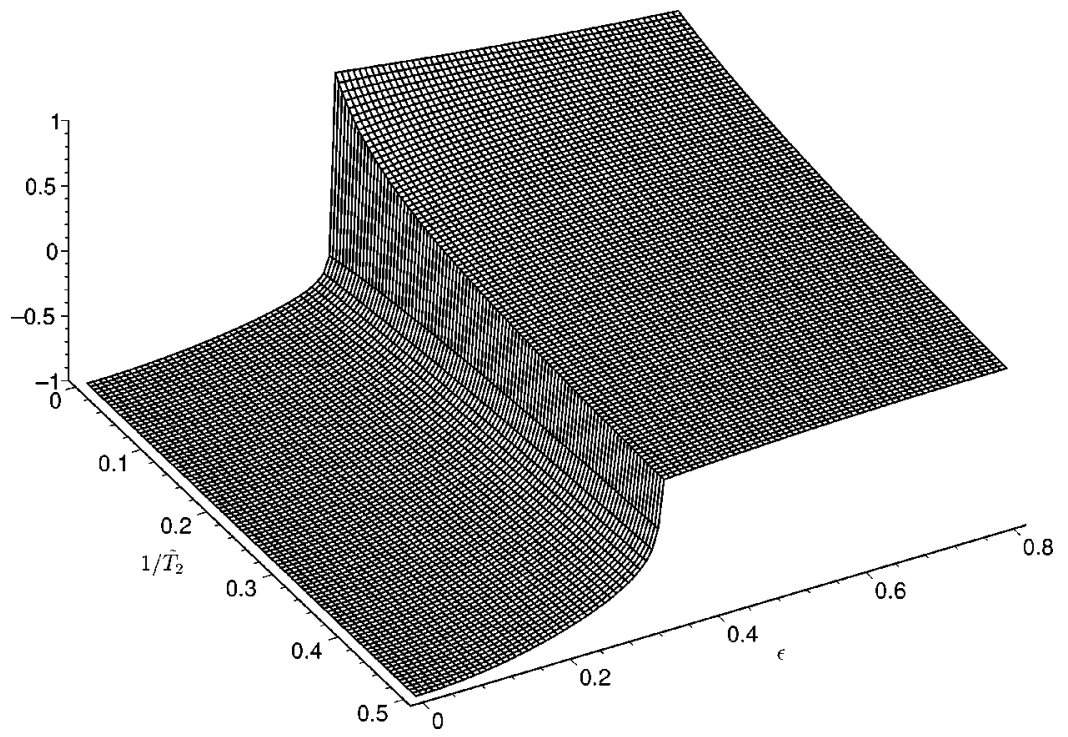

FIG. 11. Atomic population inversion $\left\langle\sigma_{3}\right\rangle$ as a function of $\epsilon / \beta_{A}$ and the nonradiative dephasing rate $1 / \widetilde{T}_{2}^{n r}$. The laser-field frequency detuning is $\Delta_{L C} / \beta_{A}=1.2, \Delta_{A L} / \beta_{A}=-1$, and $\Delta_{A C} / \beta_{A}$ $=0.2$. 
and detuning frequency. The condition that triggers this jump in the atomic population inversion corresponds to driving the left component of the atomic spectrum inside the gap, while the central resonance and the right sideband remain outside the gap.

The switching effect described here may be relevant to ultrafast all-optical switches and all-optical transistor action [7]. For this case, our analysis needs to be generalized to the case of $\mathrm{N}$-atom, non-Markovian collective switching. As a function of the intensity of the driving field (control laser), the active region of a photonic material (the atomic system) sharply switches from an absorptive medium (the atom spends most of its time in the ground state) to a gain medium (higher probability to find the atom in its excited state). A second probe laser beam will experience a substantial differential gain when the control laser intensity is in the neighborhood of the threshold value.

Our model system can be experimentally realized either by pumping a cold atomic gas in the void regions of a photonic crystals or exciting a two-level system in the dielectric region. The electric-field distribution for a laser mode in the vicinity of the upper band edge (the so-called "air" band) has strong intensity peaks in the void region of the material, which can act as a optical trap for active atoms [43]. The trapped atoms will exhibit little interaction with the lattice of the dielectric host, thus minimizing additional decay and dephasing effect. Alternatively, the radiative transition of the erbium atom at $1.537 \mu \mathrm{m}$ comes from the $4 f$ atomic shell, which is screened by the outer shells from the environmental influence. At low temperatures, the erbium atoms suitably implanted in a silicon PBG material may have very sharp single atom-like features [44] (the most intense line at 1.537 $\mu \mathrm{m}$ has a full width of $0.0005 \mu \mathrm{m})$. In this case, dephasing effects associated with the scattering of phonons on the atomic system will need to be considered.

Clearly, the experimental observation of atomic switching depends strongly on the influence of the dielectric host material on the active atoms. By including these effects in our calculations, we have shown that a sizable switching effect is present even in the presence of nonradiative decay and dephasing contribution $\left(0.08 \gamma \leqslant 1 / T_{1}^{n r}, 1 / T_{2}^{n r} \leqslant \gamma\right)$. The influence of dephasing is weaker than that of the nonradiative decay. Also, the magnitude of the driving field is extremely important. In our approximations, switching occurs when $\Omega \approx \beta$, where $\Omega$ is the generalized Rabi frequency, and $\beta$ is the typical time scale of the atomic system evolution. Since $\beta$ depends on the specific photonic crystal used, we limit ourselves to general considerations about the order of magnitude of the laser-field intensity, $I$, required to produce switching. If we neglect the detuning of the atomic frequency with respect the laser frequency, it can be easily shown that [45]

$$
\begin{aligned}
\left|\frac{\Omega}{2 \pi}[\mathrm{Hz}]\right| & =\frac{\left|\mathbf{d}_{21} \cdot \mathbf{e}_{L}\right|}{e a_{0}} \sqrt{\frac{2 e^{2} a_{0}^{2}}{\varepsilon_{0} \hbar^{2} c}} \sqrt{I\left[\mathrm{~W} / \mathrm{m}^{2}\right]} \\
& =0.2210^{7} \frac{\left|\mathbf{d}_{21} \cdot \mathbf{e}\right|}{e a_{0}} \sqrt{I\left[\mathrm{~W} / \mathrm{m}^{2}\right]} .
\end{aligned}
$$

For a typical optical transition (with dipole moments between $10^{-1}$ and $10^{1}$ atomic units) a laser field of 0.5 $\mathrm{mW} / \mathrm{mm}^{2}$ intensity will produce a Rabi frequency of 0.1 $\mathrm{GHz}$ (which is of the order of magnitude of $\beta$ ).

Our paper describes non-Markovian single-atom switching in photonic band gap materials using a second-order expansion of the equations of motion. In the Markovian evolution of a collection of two-level atoms [7] (the $\mathrm{N}$-atom generalization of Sec. IV C), an analogous collective switching effect is present. A full non-Markovian treatment of a collection of two-level atoms driven by an external field will lead to a lower threshold and much faster switching rate than discussed previously (the collective time scale factor near a three-dimensional photonic band edge has been shown to be proportional to the square of the number of atoms [7]).

\section{ACKNOWLEDGMENTS}

We are grateful to Dr. Tran Quang for a number of discussions. M.F. acknowledges support from the Natural Sciences and Engineering Research Council of Canada Scholarship Program. This work was supported in part by the Natural Sciences and Engineering Research Council of Canada and the New Energy and Industrial Technology Development Organization (NEDO) of Japan.

\section{APPENDIX A: MEMORY FUNCTIONS AND PHOTONIC CRYSTAL LOCAL DENSITY OF STATES}

The projection of the vector potential operator $\mathbf{A}$, along the direction of the atomic dipole $\hat{\mathbf{u}}_{d}$, evaluated at the center of the atom $(\mathbf{R})$, in the dipole approximation, and in the modified Coulomb gauge $(\Phi=0, \nabla \cdot[\epsilon(\mathbf{r})]=0)[46,47]$, is given by

$$
\begin{aligned}
A(\mathbf{R}) \equiv & \mathbf{A}(\mathbf{R}) \cdot \hat{\mathbf{u}}_{d} \\
= & \sum_{n, s} \int_{\mathbf{k} \in 1 \mathrm{BZ}(2 \pi)^{3 / 2}} \frac{d \mathbf{k}}{\frac{\hbar}{2 \epsilon_{0} \omega_{n, \mathbf{k}, s} \mathcal{V}}} \\
& \times\left[\mathbf{A}_{n, \mathbf{k}, s}(\mathbf{R}) \cdot \hat{\mathbf{u}}_{d}\right] a_{n, \mathbf{k}, s}+\text { H.c. }
\end{aligned}
$$

Here, $n$ is the band energy index, $s$ the polarization index, and $\mathcal{V}$ the volume of the unit cell. The $\mathbf{k}$ integration is carried over in the first Brillouin zone (1BZ). The Bloch-mode $\{n, \mathbf{k}, s\}$ annihilation (creation) operator is denoted by $a_{n, \mathbf{k}, s}$ $\left(a_{n, \mathbf{k}, s}^{\dagger}\right)$, its amplitude at the atomic location is denoted by $\mathbf{A}_{n, \mathbf{k}, s}(\mathbf{R})$, and the mode frequency by $\omega_{n, \mathbf{k}, s}$. The anisotropy of the photonic crystal lifts the polarization degeneracy, and the mode energy (frequency) acquires a supplementary index $s$. The interaction Hamiltonian between the atomic system and the radiation field reservoir is given by

$$
H=i \hbar \sum_{\lambda} g_{\lambda}(\mathbf{R}) a_{\lambda}^{\dagger} \sigma_{12}+\text { H.c. }
$$

where $\lambda$ denotes the triplet $\{n, \mathbf{k}, s\}$ and the coupling constant $g_{\lambda}(\mathbf{R})$ is 


$$
g_{n, \mathbf{k}, s}(\mathbf{R})=\frac{\omega_{A} d_{21}}{\hbar}\left[\frac{\hbar}{2 \epsilon_{0} \omega_{n, \mathbf{k}, s} \mathcal{V}}\right]^{1 / 2} \mathbf{A}_{n, \mathbf{k}, s}(\mathbf{R}) \cdot \mathbf{u}_{d}
$$

The memory function (3.3) becomes

$$
\begin{aligned}
G\left(\mathbf{R}, t-t^{\prime}\right)= & \frac{\omega_{A}^{2} d_{21}^{2}}{16 \pi^{3} \hbar \varepsilon_{0}} \sum_{n, s} \int_{\mathbf{k} \in 1 \mathrm{BZ}} d \mathbf{k} \frac{e^{-i \Delta_{n, \mathbf{k}, s}\left(t-t^{\prime}\right)}}{\omega_{n, \mathbf{k}, s}} \\
& \times\left|\mathbf{A}_{n, \mathbf{k}, s}(\mathbf{R}) \mathbf{u}_{d}\right|^{2} .
\end{aligned}
$$

Clearly, the memory function depends on the actual position of the atom in the unit cell and on the orientation of the atomic dipole with respect to the symmetry axis of the photonic crystal. However, taking into account the randomness of the atomic dipole orientation, we may average the memory function over all the possible dipole orientations. The orientation-averaged memory function is given by

$$
\begin{aligned}
G_{a v}\left(\mathbf{R}, t-t^{\prime}\right) & \equiv \frac{1}{4 \pi}\left\langle G\left(\mathbf{R}, t-t^{\prime}\right)\right\rangle_{\mathbf{u}_{d}} \\
& =\frac{\omega_{A}^{2} d_{21}^{2}}{192 \pi^{2} \hbar \varepsilon_{0}} \int_{0}^{\infty} \rho_{l o c}(\mathbf{R}, \omega) \frac{e^{-i\left(\omega-\omega_{21}\right)\left(t-t^{\prime}\right)}}{\omega^{3}} d \omega .
\end{aligned}
$$

Here, $\rho_{l o c}(\mathbf{R}, \omega)$ is the local density of states [48] defined by

$$
\rho_{l o c}(\mathbf{R}, \omega)=\sum_{n, s} \int_{\mathbf{k} \in 1 \mathrm{BZ}} d \mathbf{k} \delta\left(\omega-\omega_{n, \mathbf{k}, s}\right)\left|\mathbf{E}_{n, \mathbf{k}, s}(\mathbf{R})\right|^{2},
$$

with $\mathbf{E}_{n, \mathbf{k}, s}(\mathbf{R})=i \omega_{n, \mathbf{k}, s}(\mathbf{R}) \mathbf{A}_{n, \mathbf{k}, s}(\mathbf{R})$, the electric-field Bloch mode, $\{n, \mathbf{k}, s\}$, amplitude at the atomic position.

\section{APPENDIX B: MEMORY FUNCTIONS AND THEIR LAPLACE TRANSFORMS}

\section{Isotropic model}

Using the definition (3.3), the memory function becomes

$$
G_{I}(\tau)=\frac{\alpha}{(2 \pi)^{3}} \sum_{\mathbf{k}, \sigma}\left|\mathbf{e}_{\mathbf{k}, \sigma} \mathbf{u}_{\mathbf{d}}\right|^{2} \frac{e^{-i\left(\omega(\mathbf{k})-\omega_{A}\right) \tau}}{\omega(\mathbf{k})},
$$

where $\sigma$ represents the polarization index and $\alpha$ is given by $\alpha=\omega_{A}^{2} d_{21}^{2} / 2 \hbar \varepsilon_{0}$.

For the isotropic dispersion relation $\omega(k)=\omega_{C}+A_{I}(k$ $\left.-k_{0}\right)^{2}$, we have

$$
G_{I}(\tau)=\alpha_{I} e^{i \Delta_{A C} \tau} \int_{k_{0}}^{\infty} d k k^{2} \frac{e^{-i A_{I}\left(k-k_{0}\right)^{2} \tau}}{\omega_{C}+A_{I}\left(k-k_{0}\right)^{2}},
$$

with $\Delta_{A C}=\omega_{C}-\omega_{A}$ and $\alpha_{I}=8 \alpha / 3 \pi^{2}$. By evaluating the $k$ integral we get

$$
\begin{aligned}
G_{I}(\tau)= & \alpha_{I} e^{i \Delta_{A C} \tau}\left\{\frac { \sqrt { \omega _ { C } } \sqrt { \pi } } { 2 A _ { I } ^ { 3 / 2 } } \left[\sqrt{\pi} e^{i \omega_{C} \tau} \operatorname{Erfc}\left(\sqrt{i \omega_{C} \tau}\right)\right.\right. \\
& \left.+\frac{1}{\sqrt{i \omega_{C} \tau}}\right]+\frac{k_{0}}{A_{I}} e^{i \omega_{C} \tau} \operatorname{Ei}\left(1, i \omega_{C} \tau\right) \\
& \left.+\frac{\pi k_{0}^{2}}{2 \sqrt{\omega_{C} A_{I}}} e^{i \omega_{C} \tau} \operatorname{Erfc}\left(\sqrt{i \omega_{C} \tau}\right)\right\}
\end{aligned}
$$

Here, $\operatorname{Erfc}(x)$ is the complementary error function defined for all complex $x$ by $\operatorname{Erfc}(x)=1-\operatorname{Erf}(x)$, with $\operatorname{Erf}(x)$ $=(2 / \sqrt{\pi}) \int_{0}^{x} d t e^{-t^{2}}$. This has the asymptotic behavior $\lim _{x \rightarrow \infty} \operatorname{Erfc}(x)=(1 / \sqrt{\pi} x) e^{-x^{2}}$. Also, the exponential integral $\operatorname{Ei}(1, x)$ is defined for $\operatorname{Re}(x)>0$ by $\operatorname{Ei}(1, x)$ $=\int_{1}^{\infty} d t e^{-x t} / t$ and extended by analytic continuation to the entire complex plane (except for the branch point $x=0$ ). This has the asymptotic behavior $\lim _{x \rightarrow \infty} \operatorname{Ei}(1, x)=e^{-x} / x$.

The memory function simplifies considerably in the approximation $A \approx \omega_{C} / k_{0}^{2}$,

$$
G_{I}(\tau)=\alpha_{I} \frac{\sqrt{\omega_{C}} \sqrt{\pi}}{2 A_{I}^{3 / 2}} e^{i \Delta_{A C} \tau}\left[\sqrt{\frac{\pi}{i \omega_{C} \tau}}+2 e^{i \omega_{C} \tau} \operatorname{Ei}\left(1, i \omega_{C} \tau\right)\right],
$$

and, in the long-time limit $\left(\omega_{C} \tau \gg 1\right)$, becomes

$$
G_{I}(\tau)=\beta_{I}^{3 / 2} \frac{e^{i\left(\Delta_{C} \tau-\pi / 4\right)}}{\sqrt{\tau}},
$$

with $\quad \beta_{I}^{3 / 2}=\left(\omega_{A}^{2} d_{21}^{2} / 12 \varepsilon_{0} \hbar \pi^{3 / 2}\right)\left(k_{0}^{2} / \omega_{C} \sqrt{A_{I}}\right)$ $\approx \omega_{A}^{7 / 2} d_{21}^{2} / 6 \hbar \varepsilon_{0} \pi^{3 / 2} c^{3}$.

The Laplace transform of the isotropic model memory function can be evaluated as follows:

$$
\begin{aligned}
\widetilde{G}_{I}(p)= & \mathcal{L}\left[G_{I}(\tau)\right]=\int_{0}^{\infty} d t e^{-p t} G_{I}(\tau) \\
= & \alpha_{I} \int_{k_{0}}^{\infty} d k k^{2} \\
& \times \frac{1}{\left[\omega_{C}+A_{I}\left(k-k_{0}\right)^{2}\right]\left[p-i\left(\Delta_{A C}-A_{I}\left(k-k_{0}\right)^{2}\right)\right]} .
\end{aligned}
$$

The integral in Eq. (B6) can be evaluated by contour integration methods and the memory function becomes

$$
\begin{aligned}
\widetilde{G}_{I}(p)= & -i \frac{\alpha_{I}}{A_{I}^{2}}\left\{i \frac{\pi}{2}\left(\frac{A_{I}}{\omega_{C}}\right)^{3 / 2}\right. \\
& \times k_{0}^{2}\left[1-i \sqrt{\frac{i p+\Delta_{A C}}{\omega_{C}}}\right]^{-1} \sqrt{\frac{i p+\Delta_{A C}}{\omega_{C}}-1} \\
& -\frac{k_{0} A_{I}}{\omega_{C}}\left[\ln \left(\frac{i p+\Delta_{A C}}{\omega_{C}}\right)-i \pi\right]\left[1+\frac{i p+\Delta_{A C}}{\omega_{C}}\right]^{-1} \\
& \left.+\frac{\pi}{2} \sqrt{\frac{A_{I}}{\omega_{C}}}\left[1-i \sqrt{\frac{i p+\Delta_{A C}}{\omega_{C}}}\right]^{-1}\right\}
\end{aligned}
$$


In the long-time limit $\left(\omega_{C} \tau \gg 1, s / \omega_{C} \ll 1\right)$, the Laplace transform of the memory function (B5) becomes

$$
\widetilde{G}_{I}(p)=\beta_{I}^{3 / 2} e^{-i \pi / 4} \sqrt{\frac{\pi}{p-i \Delta_{A C}}} .
$$

\section{Anisotropic model}

In the case of an anisotropic model $\omega(\mathbf{k})=\omega_{C}+A_{I}(\mathbf{k}$ $\left.-\mathbf{k}_{0}\right)^{2}$, Eq. (B2) becomes

$$
G_{A}(\tau)=\alpha_{A} e^{i \Delta_{A C} \tau} \int_{\mathbf{k}_{0}}^{\infty} d \mathbf{k} \frac{e^{-i A_{A}\left(\mathbf{k}-\mathbf{k}_{0}\right)^{2} \tau}}{\omega_{C}+A_{A}\left(\mathbf{k}-\mathbf{k}_{0}\right)^{2}},
$$

with $\Delta_{A C}=\omega_{C}-\omega_{A}$ and $\alpha_{A}=\alpha / 2 \pi^{2}$. By evaluating the $\mathbf{k}$ space integral, we get

$$
G_{A}(\tau)=\alpha_{A} \sqrt{\frac{\omega_{C}}{A_{A}^{3}}} e^{i \Delta_{A C} \tau}\left\{\sqrt{\frac{\pi}{i \omega_{C} \tau}}-\pi e^{i \omega_{C} \tau} \operatorname{Erfc}\left[\sqrt{i \omega_{C} \tau}\right]\right\}
$$

In the long-time limit $\left(\omega_{C} \tau \gg 1\right)$, the anisotropic memory function becomes

$$
G_{A}(\tau)=-\beta_{A}^{1 / 2} \frac{e^{i\left(\Delta_{A C} \tau+\pi / 4\right)}}{\tau^{3 / 2}}
$$

with $\beta_{A}^{1 / 2}=\left(\omega_{A}^{2} d_{21}^{2} / 16 \varepsilon_{0} \hbar \pi^{3 / 2}\right)\left(1 / \omega_{C} A_{A}^{3 / 2}\right)$.

The Laplace transform of the anisotropic model memory function can be evaluated in a similar fashion:

$$
\begin{aligned}
\widetilde{G}_{A}(p)= & \mathcal{L}\left[G_{A}(\tau)\right]=\int_{0}^{\infty} d t e^{-p t} G_{A}(\tau) \\
= & \alpha_{A} \int_{\mathbf{k}_{0}}^{\infty} d \mathbf{k} \\
& \times \frac{1}{\left[\omega_{C}+A_{I}\left(\mathbf{k}-\mathbf{k}_{0}\right)^{2}\right]\left[p-i\left(\Delta_{A C}-A_{I}\left(\mathbf{k}-\mathbf{k}_{0}\right)^{2}\right)\right]} .
\end{aligned}
$$

The integral in Eq. (B12) can be evaluated by contour integration methods and the memory function becomes

$$
\widetilde{G}_{A}(p)=-i \frac{\alpha_{A} \pi}{2 \sqrt{\omega_{C}} A_{A}^{3 / 2}}\left[1-i \sqrt{\frac{i p+\Delta_{A C}}{\omega_{C}}}\right]^{-1} .
$$

In order to evaluate the long-time behavior of the Laplace transform of the anisotropic memory function, we have to deal with the nonintegrability of the memory function (B5). However, this is an artifact of the long-time expansion; the exact memory function actually possesses a much weaker (square root) singularity at $\tau=0$ and is thus integrable. In this paper, we use the regularized version introduced in $[34,43,49]$, in which the Laplace transform of anisotropic memory is given by

$$
\widetilde{G}_{A}(p)=\beta_{A}^{1 / 2} e^{i \pi / 4} \sqrt{p-i \Delta_{A C}}
$$

\section{APPENDIX C: SPONTANEOUS EMISSION, TEMPORAL EVOLUTION, AND STEADY-STATE SOLUTION}

\section{Isotropic model}

For the atomic system evolution is described by Eq. (3.6), and the atomic polarization is given by

$$
S_{21}(\tau)=\frac{1}{2 \pi i} \int_{\epsilon-i \infty}^{\epsilon+i \infty} d p e^{p \tau}\left[p+\frac{e^{-i \pi / 4}}{\sqrt{p-i \widetilde{\Delta}_{A C}}}\right]^{-1} .
$$

Here, the real number $\epsilon$ is chosen so that the line $p=\epsilon$ lies to the right of all singularities (poles and essential singularities) of the function $z(p)$, and the time and atomic detuning have been scaled so that $\tau=\beta_{I} t$ and $\widetilde{\Delta}_{A C}=\Delta_{A C} / \beta_{I}$. The inverse Laplace transform yields

$$
\begin{aligned}
S_{21}(\tau)= & e^{i \tilde{\Delta}_{A C^{t}}}\left\{\sum_{k=1}^{3} a_{k} x_{k}\left(1+r_{k}\right) e^{x_{k}^{2} \tau}\right. \\
& \left.-\sum_{k=1}^{3} a_{k} x_{k} r_{k} \operatorname{Erfc}\left(\sqrt{x_{k}^{2} \tau}\right) e^{x_{k}^{2} \tau}\right\},
\end{aligned}
$$

where

$$
\begin{gathered}
x_{1}=\left(A_{+}+A_{-}\right) e^{i \pi / 4}, \\
x_{2}=\left(A_{+} e^{-i \pi / 6}-A_{-} e^{i \pi / 6}\right) e^{-i \pi / 4}, \\
x_{3}=\left(A_{+} e^{i \pi / 6}-A_{-} e^{-i \pi / 6}\right) e^{3 i \pi / 4}, \\
A_{ \pm}=\left[\frac{1}{2} \pm \frac{1}{2}\left(1+\frac{4}{27} \widetilde{\Delta}_{A C}^{3}\right)^{1 / 2}\right]^{1 / 3}, \\
a_{k}=\frac{x_{k}}{\left(x_{k}-x_{i}\right)\left(x_{k}-x_{j}\right)}(k \neq i \neq k ; k, i, j=\overline{1,3}), \\
r_{k}=\operatorname{csgn}\left(x_{k}\right),
\end{gathered}
$$

and $\operatorname{Erfc}(x)$ is the error function [50] and $\operatorname{csgn}(x)$ is the generalized sign function for real and complex expressions, defined by

$$
\operatorname{csgn}(x)=\left\{\begin{array}{lllllll}
1 & \text { if } & \operatorname{Re}(x)>0 & \text { or } & {[\operatorname{Re}(x)=0} & \text { and } & \operatorname{Im}(x)>0] \\
-1 & \text { if } & \operatorname{Re}(x)<0 & \text { or } & {[\operatorname{Re}(x)=0} & \text { and } & \operatorname{Im}(x)<0]
\end{array}\right.
$$


The value of $r_{2}$ is determined by the atomic detuning $\widetilde{\Delta}_{A C}$ :

$$
r_{2}= \begin{cases}1 & \text { if } \tilde{\Delta}_{A C} \geqslant-3 a /\left[1+a^{3}\right]^{2 / 3}, \\ -1 & \text { otherwise, }\end{cases}
$$

where $a=2+\sqrt{3}, r_{1}=1$, and $r_{3}=-1$ are independent of the value of the atomic detuning. The temporal behavior of the atomic operator $\sigma_{21}$ is then given by

$$
\begin{aligned}
\sigma_{21}(\tau)= & \sigma_{21}(0) e^{i \widetilde{\Delta}_{A C} \tau}\left\{2 a_{1} x_{1} e^{x_{1}^{2} \tau}+a_{2} x_{2}\left(1+r_{2}\right) e^{x_{2}^{2} \tau}\right. \\
& \left.-\sum_{k=1}^{3} a_{k} x_{k} r_{k} \operatorname{Erfc}\left(\sqrt{x_{k}^{2} \tau}\right) e^{x_{k}^{2} \tau}\right\}
\end{aligned}
$$

For large time, the terms of higher order than $\tau^{-3 / 2}$ can be neglected, and Eq. (C9) reduces to

$$
\begin{aligned}
\sigma_{21}(\tau)= & \sigma_{21}(0) e^{i \tilde{\Delta}_{A C} \tau}\left\{2 a_{1} x_{1} e^{x_{1}^{2} \tau}+a_{2} x_{2}\left(1+r_{2}\right) e^{x_{2}^{2} \tau}\right. \\
& \left.+\frac{1}{2 \sqrt{\pi}}\left[\sum_{k=1}^{3} \frac{a_{k}}{x_{k}^{2}}\right] \frac{1}{\tau^{3 / 2}}\right\}
\end{aligned}
$$

The formal solution of the excited atomic population is

$$
\begin{aligned}
S_{22}(\tau)= & \frac{1}{2 \pi i} \int_{\epsilon-i \infty}^{\epsilon+i \infty} d p e^{p \tau}\left[p+\frac{e^{-i \pi / 4}}{\sqrt{p-i \widetilde{\Delta}_{A C}}}\right. \\
& \left.+\frac{e^{i \pi / 4}}{\sqrt{p+i \tilde{\Delta}_{A C}}}\right]^{-1} .
\end{aligned}
$$

Since the Laplace inversion of Eq. (C11) requires the formal solution of a sixth-order polynomial equation, we opt for a numerical approach. For each value of the atomic detuning, we numerically find the singularities of $x(p)$, which are then used in an Adams algorithm [39], to perform the Laplace transform inversion.

\section{Anisotropic model}

By scaling the time and frequency $\tau \equiv \beta_{A} t$ and $\widetilde{\Delta}_{A C}$ $\equiv \Delta_{A C} / \beta_{A}$, the formal solutions for the atomic population and polarization become

$$
\begin{gathered}
S_{22}(\tau)=\frac{1}{2 \pi i} \int_{\epsilon-i \infty}^{\epsilon+i \infty} d p e^{p \tau}\left[p+\beta_{A}^{1 / 2}\left(e^{i \pi / 4} \sqrt{p-i \Delta_{A C}}\right.\right. \\
\left.\left.+e^{-i \pi / 4} \sqrt{p+i \Delta_{A C}}\right)\right]^{-1}, \\
S_{21}(\tau)=\frac{1}{2 \pi i} \int_{\epsilon-i \infty}^{\epsilon+i \infty} d p e^{p \tau}\left[p+\beta_{A}^{1 / 2} e^{i \pi / 4} \sqrt{p-i \Delta_{A C}}\right]^{-1}
\end{gathered}
$$

The inverse Laplace transform of $z(p)$ yields

$$
\begin{aligned}
S_{21}(\tau)= & e^{i \widetilde{\Delta}_{A C^{t}}}\left\{\sum_{k=1}^{2} b_{k} x_{k}\left(1+r_{k}\right) e^{x_{k}^{2} \tau}\right. \\
& \left.-\sum_{k=1}^{3} b_{k} x_{k} r_{k} \operatorname{Erfc}\left(\sqrt{x_{k}^{2} \tau}\right) e^{x_{k}^{2} \tau}\right\},
\end{aligned}
$$

where

$$
\begin{gathered}
x_{1,2}=-\frac{\sqrt{i}}{2}\left[1 \pm \sqrt{1-4 \widetilde{\Delta}_{A C}}\right], \\
b_{k}=\frac{1}{x_{i}-x_{j}}(k \neq i \neq j ; k, i, j=1,2), \\
r_{k}=\operatorname{csgn}\left(x_{k}\right) .
\end{gathered}
$$

The value of $r_{1}$ is determined by the atomic detuning $\widetilde{\Delta}_{A C}$ and one can show that

$$
r_{1}=\left\{\begin{array}{rrrr}
1 & \text { if } \quad \tilde{\Delta}_{A C} & \in & (-\infty, 0) \cup\left(\frac{1}{2}, \infty\right), \\
-1 & \text { if } \quad \tilde{\Delta}_{A C} & \in & \left(0, \frac{1}{2}\right),
\end{array}\right.
$$

while $r_{2}=-1$ is independent of the value of the atomic detuning frequency $\widetilde{\Delta}_{A C}$. The expression of the atomic operator $\sigma_{21}$ is then given by

$$
\begin{aligned}
\sigma_{21}(\tau)= & \sigma_{21}(0) e^{i \widetilde{\Delta}_{A C} \tau}\left\{2 b_{1} x_{1} e^{x_{1}^{2} \tau}\right. \\
& \left.-\sum_{k=1}^{2} b_{k} x_{k} r_{k} \operatorname{Erfc}\left(\sqrt{x_{k}^{2} \tau}\right) e^{x_{k}^{2} \tau}\right\} .
\end{aligned}
$$

\section{APPENDIX D: NONRADIATIVE RELAXATION AND DEPHASING EFFECTS}

In order to compare our model to experiments, we include phenomenological decay rates $1 / T_{1}^{n r}$ and $1 / T_{2}^{n r}$ associated with other (nonradiative) decay and dephasing, respectively.

In the bare picture, Eqs. (4.4b) and (4.4c) become

$$
\begin{gathered}
\frac{d}{d t} \sigma_{21}(t)=\Lambda \eta^{\dagger}(t) \sigma_{3}(t)-i \varepsilon \sigma_{3}(t)+\left(i \Delta_{A L}-\frac{1}{T_{2}^{n r}}\right) \sigma_{21}(t) \\
+\Lambda^{2} \int_{0}^{t} d t^{\prime} G\left(t-t^{\prime}\right) \sigma_{21}\left(t^{\prime}\right) \sigma_{3}(t) \\
\frac{d}{d t} \sigma_{3}(t)=-2 \Lambda \sigma_{21}(t) \eta(t)-2 i \varepsilon \sigma_{21}(t) \\
-2 \Lambda^{2} \int_{0}^{t} d t^{\prime} G\left(t-t^{\prime}\right) \sigma_{21}(t) \sigma_{12}\left(t^{\prime}\right) \\
-\frac{1}{2 T_{1}^{n r}}\left(\sigma_{3}+1\right)+\text { H.c. }
\end{gathered}
$$

Using the dressed-state transformation (4.5), the equations of motion are given by 


$$
\begin{aligned}
\left\langle\frac{d}{d t} R_{21}(t)\right\rangle= & -2 c^{2} s^{2} \Lambda^{2} \int_{0}^{t} d t^{\prime} G_{0}^{*}\left(t-t^{\prime}\right)\left\langle R_{21}\left(t^{\prime}\right)\right\rangle-c^{4} \Lambda^{2} \int_{0}^{t} d t^{\prime} G_{+}^{*}\left(t-t^{\prime}\right)\left\langle R_{21}\left(t^{\prime}\right)\right\rangle-2 c^{2} s^{2} \Lambda^{2} \int_{0}^{t} d t^{\prime} G_{0}\left(t-t^{\prime}\right)\left\langle R_{21}\left(t^{\prime}\right)\right\rangle \\
& -s^{4} \Lambda^{2} \int_{0}^{t} d t^{\prime} G_{-}\left(t-t^{\prime}\right)\left\langle R_{21}\left(t^{\prime}\right)\right\rangle-\left[\frac{1}{T_{2}^{n r}}\left(s^{4}+c^{4}\right)+2 \frac{1}{T_{1}^{n r}} s^{2} c^{2}\right]\left\langle R_{21}(t)\right\rangle+2 e^{-2 i \Omega t}\left\{c s^{3} \Lambda^{2} \int_{0}^{t} d t^{\prime} G_{-}^{*}\left(t-t^{\prime}\right)\right. \\
& \times\left\langle R_{11}\left(t^{\prime}\right)\right\rangle+c^{3} s \Lambda^{2} \int_{0}^{t} d t^{\prime} G_{+}\left(t-t^{\prime}\right)\left\langle R_{22}\left(t^{\prime}\right)\right\rangle+\frac{c^{3} s}{2} \Lambda^{2} \int_{0}^{t} d t^{\prime} G_{0}^{*}\left(t-t^{\prime}\right)+\frac{c s^{3}}{2} \Lambda^{2} \int_{0}^{t} d t^{\prime} G_{0}\left(t-t^{\prime}\right) \\
& \left.-\frac{s c\left(s^{2}-c^{2}\right)}{2}\left(\frac{1}{T_{1}^{n r}}-\frac{1}{T_{2}^{n r}}\right)\left\langle R_{3}(t)\right\rangle+\frac{s c}{2 T_{1}^{n r}}\right\}-e^{-4 i \Omega t}\left\{c^{2} s^{2} \Lambda^{2} \int_{0}^{t} d t^{\prime} G_{-}^{*}\left(t-t^{\prime}\right)\left\langle R_{12}\left(t^{\prime}\right)\right\rangle\right. \\
& \left.+c^{2} s^{2} \Lambda^{2} \int_{0}^{t} d t^{\prime} G_{+}\left(t-t^{\prime}\right)\left\langle R_{12}\left(t^{\prime}\right)\right\rangle-s^{2} c^{2}\left(\frac{1}{T_{1}^{n r}}-\frac{1}{T_{2}^{n r}}\right)\left\langle R_{12}(t)\right\rangle\right\}, \\
\left\langle\frac{d}{d t} R_{3}(t)\right\rangle=- & 2 c^{4} \Lambda^{2} \int_{0}^{t} d t^{\prime} G_{+}\left(t-t^{\prime}\right)\left\langle R_{22}\left(t^{\prime}\right)\right\rangle+2 s^{4} \Lambda^{2} \int_{0}^{t} d t^{\prime} G_{-}\left(t-t^{\prime}\right)\left\langle R_{11}\left(t^{\prime}\right)\right\rangle-\left[\frac{2 s^{2} c^{2}}{T_{2}^{n r}}+\frac{\left(c^{2}-s^{2}\right)^{2}}{2 T_{1}^{n r}}\right]\left\langle R_{3}(t)\right\rangle+\frac{s^{2}-c^{2}}{2 T_{1}^{n r}} \\
- & 2 c s^{3} e^{-2 i \Omega t} \Lambda^{2} \int_{0}^{t} d t^{\prime} G_{0}\left(t-t^{\prime}\right)\left\langle R_{12}\left(t^{\prime}\right)\right\rangle-s c\left(c^{2}-s^{2}\right) e^{-2 i \Omega t}\left(\frac{1}{T_{1}^{n r}}-\frac{1}{T_{2}^{n r}}\right)\left\langle R_{12}(t)\right\rangle \\
+ & 2 c^{3} s e^{2 i \Omega t} \Lambda^{2} \int_{0}^{t} d t^{\prime} G_{0}\left(t-t^{\prime}\right)\left\langle R_{21}\left(t^{\prime}\right)\right\rangle-s c\left(c^{2}-s^{2}\right) e^{2 i \Omega t}\left(\frac{1}{T_{1}^{n r}}-\frac{1}{T_{2}^{n r}}\right)\left\langle R_{21}(t)\right\rangle+\mathrm{H} . \mathrm{c} .
\end{aligned}
$$

We now consider certain special cases of these general equations. If the external laser field is sufficiently strong that the secular approximation is justified, the evolution of the atomic system is given by

$$
\begin{gathered}
\frac{d}{d t}\left\langle R_{21}(t)\right\rangle=-2 c^{2} s^{2} \int_{0}^{t} d t^{\prime} G_{0}^{*}\left(t-t^{\prime}\right)\left\langle R_{21}\left(t^{\prime}\right)\right\rangle-c^{4} \int_{0}^{t} d t^{\prime} G_{+}^{*}\left(t-t^{\prime}\right)\left\langle R_{21}\left(t^{\prime}\right)\right\rangle-2 c^{2} s^{2} \int_{0}^{t} d t^{\prime} G_{0}\left(t-t^{\prime}\right)\left\langle R_{21}\left(t^{\prime}\right)\right\rangle \\
-s^{4} \int_{0}^{t} d t^{\prime} G_{-}\left(t-t^{\prime}\right)\left\langle R_{21}\left(t^{\prime}\right)\right\rangle-\left[\frac{1}{T_{2}^{n r}}\left(s^{4}+c^{4}\right)+2 \frac{1}{T_{1}^{n r}} s^{2} c^{2}\right]\left\langle R_{21}(t)\right\rangle, \\
\frac{d}{d t}\left\langle R_{3}(t)\right\rangle=-2 c^{4} \int_{0}^{t} d t^{\prime} G_{+}\left(t-t^{\prime}\right)\left\langle R_{22}\left(t^{\prime}\right)\right\rangle+2 s^{4} \int_{0}^{t} d t^{\prime} G_{-}\left(t-t^{\prime}\right)\left\langle R_{11}\left(t^{\prime}\right)\right\rangle-\left[\frac{2 s^{2} c^{2}}{T_{2}^{n r}}+\frac{\left(c^{2}-s^{2}\right)^{2}}{2 T_{1}^{n r}}\right]\left\langle R_{3}(t)\right\rangle+\frac{s^{2}-c^{2}}{2 T_{1}^{n r}}+\text { H.c. }
\end{gathered}
$$

In the case of a strong external laser field and Markovian approximation, considered in Sec. IV C, the temporal evolution of the dressed atomic polarization and inversion [Eqs. (4.11)] is given by

$$
\begin{gathered}
\left\langle R_{21}(t)\right\rangle=\left\langle R_{21}(0)\right\rangle \exp \left[-\frac{1}{2}\left(4 c^{2} s^{2} \gamma_{0}+c^{4} \gamma_{+}+s^{4} \gamma_{-}+\frac{s^{4}+c^{4}}{T_{2}^{n r}}+2 \frac{s^{2} c^{2}}{T_{1}^{n r}}\right) t\right], \\
\left\langle R_{3}(t)\right\rangle=\left\langle R_{3}(0)\right\rangle \exp \left[-\left(c^{4} \gamma_{+}+s^{4} \gamma_{-}+4 \frac{s^{2} c^{2}}{T_{2}^{n r}}+\frac{\left(s^{2}-c^{2}\right)^{2}}{T_{1}^{n r}}\right) t\right] \\
+\frac{c^{4} \gamma_{+}-s^{4} \gamma_{-}+\left(s^{2}-c^{2}\right) / T_{1}^{n r}}{c^{4} \gamma_{+}+s^{4} \gamma_{-}+4 s^{2} c^{2} / T_{2}^{n r}+\left(s^{2}-c^{2}\right)^{2} / T_{1}^{n r}}\left\{1-\exp \left[-\left(c^{4} \gamma_{+}+s^{4} \gamma_{-}+4 \frac{s^{2} c^{2}}{T_{2}^{n r}}+\frac{\left(s^{2}-c^{2}\right)^{2}}{T_{1}^{n r}}\right) t\right]\right\} .
\end{gathered}
$$

In the non-Markovian case (Sec. IV D), when the spectral features are placed in the neighborhood of a photonic band edge, the Laplace transforms of the dressed atomic inversion and polarization become [Eqs. (4.12a) and (4.12b)] 


$$
\begin{gathered}
x(p)=\frac{x_{0} p+\left[s^{4} \widetilde{G}_{-}(p)-c^{4} \widetilde{G}_{+}(p)+\left(s^{2}-c^{2}\right) / 2 T_{1}^{n r}+\text { c.c. }\right]}{p\left\{p+\left[c^{4} \widetilde{G}_{+}(p)+s^{4} \widetilde{G}_{-}(p)+2 s^{2} c^{2} / T_{2}^{n r}+\left(s^{2}-c^{2}\right)^{2} / 2 T_{1}^{n r}+\text { c.c. }\right]\right\}}, \\
z(p)=\frac{z_{0}}{p+c^{4} \widetilde{G}_{+}^{*}(p)+s^{4} \widetilde{G}_{-}(p)+2 c^{2} s^{2}\left[\widetilde{G}_{0}(p)+\widetilde{G}_{0}^{*}(p)\right]+\left(s^{4}+c^{4}\right) / T_{2}^{n r}+2 s^{2} c^{2} / T_{1}^{n r}} .
\end{gathered}
$$

For an anisotropic photonic band gap material (Sec. IV D), characterized by the photon density of states (2.3), the functions $\widetilde{G}_{0}(p), \widetilde{G}_{ \pm}(p)$ are given by Eq. (4.13). Equation (4.18) then follows from the identity $\lim _{t \rightarrow \infty}\left\{\left\langle R_{3}(t)\right\rangle\right\}=\lim _{p \rightarrow 0}\{p x(p)\}$.

[1] S. John, Phys. Rev. Lett. 53, 2169 (1984).

[2] K. M. Ho, C. T. Chan, and C. M. Soukoulis, Phys. Rev. Lett. 65, 3152 (1990).

[3] E. Yablonovitch, T. J. Gmitter, and K. M. Leung, Phys. Rev. Lett. 67, 2295 (1991).

[4] E. Yablonovitch, Phys. Rev. Lett. 58, 2059 (1987).

[5] S. John, Phys. Rev. Lett. 58, 2486 (1987).

[6] S. John and T. Quang, Phys. Rev. A 50, 1764 (1994); A. Kaufman, G. Kurizki, and B. Sherman, J. Mod. Opt. 41, 353 (1994); M. Lewenstein, J. Zakrzewski, and T. W. Mossberg, Phys. Rev. A 38, 808 (1988); Y. Yang and S. Y. Zhu, ibid. 62, 013805 (2000).

[7] S. John and T. Quang, Phys. Rev. Lett. 78, 1888 (1997).

[8] Confined Electrons and Photons, edited by E. Burstein and C. Weisbuch (Plenum, New York, 1995).

[9] S. Haroche and D. Kleppner, Phys. Today 42(1), 24 (1989).

[10] S. D. Brorson, H. Yokoyoma, and E. P. Ippen, IEEE J. Quantum Electron. 26, 1492 (1990).

[11] D. Kleppner, Phys. Rev. Lett. 47, 233 (1981).

[12] S. John and J. Wang, Phys. Rev. Lett. 64, 2418 (1990); Phys. Rev. B 43, 12772 (1991).

[13] N. Vats and S. John, Phys. Rev. A 58, 4168 (1998).

[14] S. John and T. Quang, Phys. Rev. A 54, 4479 (1996).

[15] H. Charmichael, An Open Systems Approach to Quantum Optics (Springer-Verlag, Berlin, 1993).

[16] K. Wódkiewicz and J. H. Eberly, Ann. Phys. (N.Y.) 101, 574 (1976).

[17] M. Lewenstein, T. W. Mossberg, and R. J. Glauber, Phys. Rev. Lett. 59, 775 (1987).

[18] M. Lewenstein and T. W. Mossberg, Phys. Rev. A 37, 2048 (1988).

[19] L. A. Khaflin, Zh. Éksp. Teor. Fiz. 33, 1371 (1957) [Sov. Phys. JETP 6, 1053 (1958)]; L. Fonda and G. C. Ghirardi, Nuovo Cimento A 7, 180 (1972).

[20] R. Paley and N. Wiener, AMS Colloquim Publications Vol. 19 (1934).

[21] P. L. Knight and P. W. Milonni, Phys. Lett. 56A, 275 (1976).

[22] C. R. Stroud, Jr., Ph.D. thesis, Washington University, St. Louis, MO, 1969.

[23] J. Mostowski and K. Wódkiewicz, Bull. Acad. Pol. Sci., Ser. Sci., Math., Astron. Phys. 21, 1027 (1973).

[24] G. Weinrech, Solids (Wiley, New York, 1965).

[25] E. Yablonovitch, T. J. Gmitter, and K. M. Leung, Phys. Rev. Lett. 67, 2295 (1991).

[26] K. M. Ho et al., Solid State Commun. 89, 413 (1993).
[27] S. Y. Lin et al., Nature (London) 394, 251 (1998).

[28] J. E. G. L. Winjnhoven, W. L. Vos, Science 281, 802 (1998).

[29] H. Miguez et al., Phys. Rev. B 59, 1563 (1999).

[30] Y. A. Vlasov, N. Yao, and D. J. Norris, Adv. Mater. 11, 165 (1999).

[31] A. Blanco et al., Nature (London) (London) 405, 437 (2000).

[32] V. Bǔzek et al., Phys. Rev. A 60, 582 (1999).

[33] M. O. Scully and M. S. Zubairy, Quantum Optics (Cambridge University Press, Cambridge, England, 1997).

[34] S. John and T. Quang, Phys. Rev. Lett. 74, 3419 (1995).

[35] G. M. Moy, J. J. Hope, and C. M. Savage, Phys. Rev. A 59, 667 (1999).

[36] C. K. Law and J. H. Eberly, Phys. Rev. A 43, 6337 (1991); N. W. Ashcroft and N. D. Mermin, Solid State Physics (Saunders College, Philadelphia, 1976).

[37] T. Quang, Phys. Rev. A 43, 2561 (1991).

[38] L. M. Narducci, D. H. Feng, R. Gilmore, and G. S. Agarwal, Phys. Rev. A 18, 1571 (1978).

[39] A. Murli and M. Rizzardi, ACM TOMS 16, 158 (1990); A.H.D. Cheng, P. Sidauruk, and Y. Abousleiman, Math. J. 4, 56 (1994).

[40] C. J. Savant, Fundamentals of the Laplace Transformation (McGraw-Hill, New York, 1962).

[41] S. M. Barnett and P. M. Radmore, Methods in Theoretical Quantum Optics (Oxford University Press, Oxford, 1997).

[42] E. Paspalakis, N. J. Kylstra, and P. L. Knight, Phys. Rev. A 60, R33 (1999).

[43] M. Woldeyohannes and S. John, Phys. Rev. A 60, 5046 (1999).

[44] S. Lanzerstorter et al., Appl. Phys. Lett. 72, 809 (1998); V. F. Masterov et al., ibid. 72, 728 (1998); Xinwei Zhao et al., ibid. 74, 120 (1999); S. Komuro et al., ibid. 74, 377 (1999).

[45] B. W. Shore, The Theory of Coherent Atomic Excitation (Wiley, New York, 1990).

[46] G. Kweon and N. M. Lawandy, Opt. Commun. 118, 388 (1995).

[47] K. Busch, N. Vats, S. John, and B. C. Sanders, Phys. Rev. E 62, 4251 (2000); N. Vats, K. Busch, and S. John (unpublished).

[48] K. Busch and S. John, Phys. Rev. E 58, 3896 (1998).

[49] T. Quang et al., Phys. Rev. Lett. 79, 5238 (1997).

[50] I. S. Gradshteyn and I. M. Ryzhik, Table Integral, Series, and Products (Academic, New York, 1980).

[51] H.C. Torrey, Phys. Rev. 76, 1059 (1949).

[52] B.R. Mollow, Phys. Rev. A 12, 1919 (1975).

[53] W. Heitler, The Quantum Theory of Radiation (Oxford University Press, London, 1954). 